\title{
Lagrangian transport induced by peristaltic pumping in a closed channel
}

\author{
Chiu-On $\mathrm{Ng}^{*}$ and Ye Ma \\ Department of Mechanical Engineering, The University of Hong Kong, Pokfulam Road, Hong Kong, People's Republic of China
}

(Received 25 August 2009; published 16 November 2009)

\begin{abstract}
Lagrangian transport induced by peristaltic waves traveling on the boundaries of a two-dimensional rectangular closed channel is studied analytically. Based on the Lagrangian description, an asymptotic analysis is performed to generate explicit expressions for the leading-order oscillatory as well as the higher-order timemean mass transport (or steady streaming) velocities as functions of the wave properties. Two cases are considered. The first case, which is for slow wave frequency or very small wave amplitude such that the steady-streaming Reynolds number $\left(\mathrm{Re}_{s}\right)$ is very small, recovers the one studied previously in the literature, but with all the results fully presented in the Lagrangian sense. The second case, corresponding to highfrequency pumping such as $\operatorname{Re}_{s}$ is order unity, is where it has been handled analytically. It is found that the overall mixing resulting from the mass transport can depend on the phase shift of the two waves, the wave number, the frequency, as well as the amplitude of the waves.
\end{abstract}

DOI: 10.1103/PhysRevE.80.056307

PACS number(s): 47.61.Fg, 47.10.A-, 47.15.Rq

\section{INTRODUCTION}

Peristalsis, a term normally used in the context of biological transport, refers to the process in which contents in a tube are pushed forward by a series of radial contraction and relaxation of the tube wall propagating like a periodic wave down the tube. Peristaltic pumping is the driving mechanism responsible for the flow and transport in many parts of a human body, e.g., the gastrointestinal tract and the ureter. Studies on peristaltic transport in biofluid systems are very extensive; classical works include Barton and Raynor [1], Fung and Yih [2], Jaffrin and Shapiro [3], Yin and Fung [4], and many others.

In recent years, peristalsis finds its applications in microelectromechanical systems (MEMS) as well. To produce fluid mixing in a microscale system without using internal moving mechanical parts, one convenient way is to apply oscillatory transverse motions to the boundaries of the system. Selverov and Stone [5] and Yi et al. [6] are among the first who developed theories to describe such peristaltically driven microchannel flow. Their problems were to examine time-periodic as well as steady flows induced by transversely oscillating boundaries in a closed two-dimensional rectangular cavity. Particular attention was given to the limits of small wave amplitude and high wave frequency, which are representative of MEMS applications. Carlsson et al. [7,8] and Lee et al. [9] also presented theories on the steady streaming and mixing due to standing wall vibration in a channel. Numerical simulations of peristaltic species mixing in a channel with finite boundary displacements were performed by Kumar et al. [10]. The effect of slip boundary conditions on peristaltic pumping in a rectangular microchannel was recently investigated by Mandviwalla and Archer [11].

The present work aims to revisit the problem considered by Selverov and Stone [5] and Yi et al. [6], while following

\footnotetext{
*Author to whom correspondence should be addressed. cong@hku.hk
}

a different approach of analysis that enables a wider scope of analytical validity of the theory. The basic problem is examine the steady Lagrangian drift (also known as steady streaming as in acoustics, or mass transport as in water waves) of a viscous Newtonian fluid contained in a closed twodimensional cavity under the action of small-amplitude progressive peristaltic waves applied to the boundaries of the cavity. Based on the condition that the wave amplitude is much smaller than the channel depth, a perturbation analysis can be performed so that the first-order solutions are purely first harmonics in time, while the time-independent solutions are obtained in the higher orders.

This work is essentially different from Selverov and Stone [5] and Yi et al. [6] in the following aspects. First, these authors based their analyses on the Eulerian system, although they also considered Lagrangian particle transport. Here, the problem is formulated directly in terms of Lagrangian coordinates giving solutions that are Lagrangian in nature. This saves the algebra required to convert the steady-streaming velocity from the Eulerian to the Lagrangian descriptions. Second, in many parts of their deductions, the expressions for coefficients were so lengthy and cumbersome that these authors had to omit presenting these expressions in their papers. Here, each and every coefficient is fully expressed and all the results are explicitly given in a form as compact as possible. This paper is self-contained. Applying the present theory is very convenient and straightforward, without the need to use specialized packages such as MATHEMATICA or MAPLE. Third, their analytical models are constrained by the condition that the wave amplitude $a$ must be much smaller than the Stokes layer thickness $\delta=(2 \nu / \sigma)^{1 / 2}$, where $\nu$ is the kinematic viscosity of fluid and $\sigma$ is the angular frequency of the wave. This condition, which amounts to a very small steady-streaming Reynolds number, $\operatorname{Re}_{s} \equiv(a / \delta)^{2} \ll 1$, results from, among others, the Taylor expansion of a moving boundary about its mean position, as required on the imposition of a boundary condition on a moving boundary in the Eulerian approach. The solution under this condition, known as the conduction solution in water waves [12], is applicable only when the wave amplitude is extremely small, particularly when the frequency is high. In this work, the theory is 
extended to the much desired range $\operatorname{Re}_{s}=O(1)$, for which convection and viscous diffusion are equally important in the transport of second-order vorticity from the Stokes boundary layers into the inviscid core of the flow field. The present analytical model for Lagrangian mass transport, valid to $\operatorname{Re}_{s}=O(1)$, is original in the literature. Fourth, with their numerical simulations, Yi et al. [6] also considered the cases of fairly large amplitudes of boundary displacement. The way they obtained the Lagrangian particle transport was, however, rather tedious, time consuming, and computationally demanding. They had to compute the trajectories of many particles by numerically integrating with time the velocities yielded from the numerical simulation. In sharp contrast, the present model is purely analytical, with every expression explicitly given here. Calculations based on the present model are fast and easy.

This paper is organized as follows. Our problem formulated in terms of Lagrangian coordinates is explained in further detail in Sec. II, where the small ratio of wave amplitude to channel depth is used as an ordering parameter for the problem. On expanding the equations and variables in terms of the small parameter, a perturbation analysis is performed, as detailed in the following sections. In Sec. III, the firstorder problem is solved, and the solutions consist of horizontally periodic as well as horizontally uniform time-oscillatory components. In Sec. IV, the second-order problem is presented, and the focus is on the time-mean motions. Again, there are two components of the steady streaming: onedimensional and cellular components, arising from interactions between different components of the first-order oscillatory motions. In Sec. V, the case of fast oscillation such that $\operatorname{Re}_{s}=O(1)$ is looked into. This is a case in which the flow domain is divided into the boundary layers and the core region. Near the two channel walls are the thin Stokes boundary layers in which the second-order steady vorticity is generated. The transport of the vorticity into the core region is accomplished by means of convection as well as viscous diffusion in the present case where the wave amplitude is comparable with the Stokes layer thickness. To this end, the steady-streaming velocity has to be found in the fourth-order problem requiring the second- and third-harmonic solutions to be derived first. It turns out that, for this problem, only the cellular component of the steady streaming is affected by a finite value of $\mathrm{Re}_{s}$. Finally, some discussions on the mixing effect arising from the Lagrangian transport are presented in Sec. VI.

\section{PROBLEM FORMULATION}

We consider flow induced by peristaltic pumping of a viscous fluid contained in a closed rectangular channel of depth $h$ and length $L$. The channel is very wide in the spanwise direction, and hence the flow is essentially two dimensional. By Lagrangian description, the instantaneous horizontal and vertical positions of a fluid particle $(x, y)$ are functions of the undisturbed coordinates $(\alpha, \beta)$ and time $t$, where the $\alpha$ axis is along the bottom of the channel in the direction of the traveling waves and the $\beta$ axis points upward across the channel; see Fig. 1 . The origin $\alpha=0$ is set at a

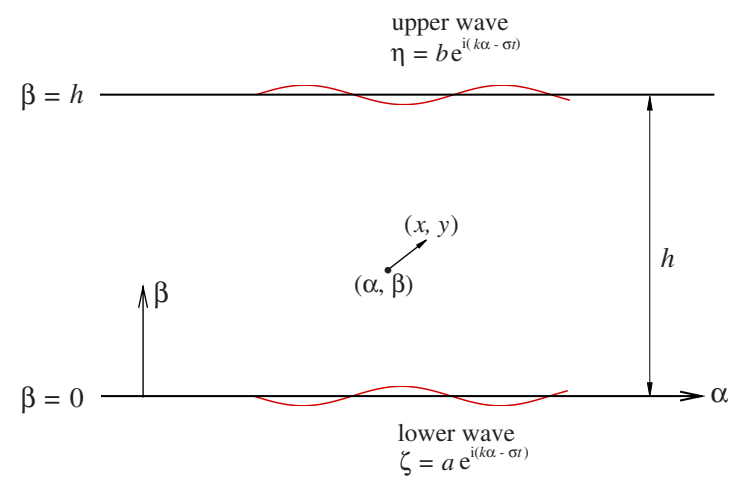

FIG. 1. (Color online) Definition sketch of peristaltic waves traveling on the upper and lower boundaries of a two-dimensional channel, where $(\alpha, \beta)$ and $(x, y)$, the Lagrangian and Eulerian coordinates, are the undisturbed and instantaneous positions of a particle.

point in the middle of the channel, and the two sidewalls are located at $\alpha=-L_{1}$ and $\alpha=L_{2}=L-L_{1}$. More is said about the origin relative to the sidewalls below.

Fluid motion is caused by prescribed transverse displacements of the two bounding walls at $\beta=0$ and $\beta=h$ in the form of traveling waves:

$$
\begin{aligned}
& y(\alpha, 0, t)=\zeta(\alpha, t)=\operatorname{Re}\left[a e^{i(k \alpha-\sigma t)}\right], \\
& y(\alpha, h, t)=\eta(\alpha, t)=\operatorname{Re}\left[b e^{i(k \alpha-\sigma t)}\right],
\end{aligned}
$$

where Re denotes the real part, $i$ is the complex unit, $a$ and $b$ are the amplitudes, $k$ is the wave number, and $\sigma$ is the angular frequency of the waves. All these wave properties are known quantities. The upper and lower waves have the same wavelength $\ell=2 \pi / k$ and period $T=2 \pi / \sigma$, but may have a different amplitude and/or phase shift. Without loss of generality, we shall take $a, k$, and $\sigma$ as real constants, and $b$ is left as a complex parameter in general.

The wall motion must be constrained near the two ends of the wall where it is anchored. To account for this, we may modify the functions $\zeta$ and $\eta$ by applying to them the factors $\left(1-\exp \left[-\left(\alpha+L_{1}\right) / m\right]\right)$ and $\left(1-\exp \left[\left(\alpha-L_{2}\right) / m\right]\right)$, where $m$ represents the stiffness of the anchor [6]. Here, very stiff anchorage is assumed so that $m$ is very small. For $m \ll \ell$, the anchoring effect can be ignored except very near the side boundaries. Yi et al. have also shown that volume conservation is satisfied only when

$$
\tan (k L / 2)=k m \tanh (L / 2 m) .
$$

In the limit $m \rightarrow 0$, which is assumed here, the equation above requires that the channel length must be an integer multiple of the wavelength, $L=n \ell$.

It is further assumed that the wavelength is comparable with the channel depth, i.e., $k h=O(1)$, and is much larger than the wave amplitudes. The small wave amplitude ratio

$$
\epsilon \equiv a / h \ll 1
$$

will be used as the ordering parameter in the following analysis. The equations of motion and boundary conditions in Lagrangian form are obtainable from Pierson [13] and are 
recalled as follows. Using the standard notation for the Jacobian, the mass conservation equation is

$$
\frac{\partial(x, y)}{\partial(\alpha, \beta)}=1,
$$

and the $x$ - and $y$-momentum equations are

$$
\begin{aligned}
& \ddot{x}=-\frac{1}{\rho} \frac{\partial(p, y)}{\partial(\alpha, \beta)}+\frac{1}{\rho}\left[\frac{\partial\left(\tau_{x x}, y\right)}{\partial(\alpha, \beta)}+\frac{\partial\left(x, \tau_{x y}\right)}{\partial(\alpha, \beta)}\right], \\
& \ddot{y}=-\frac{1}{\rho} \frac{\partial(x, p)}{\partial(\alpha, \beta)}+\frac{1}{\rho}\left[\frac{\partial\left(\tau_{y x}, y\right)}{\partial(\alpha, \beta)}+\frac{\partial\left(x, \tau_{y y}\right)}{\partial(\alpha, \beta)}\right],
\end{aligned}
$$

and the stress components are

$$
\begin{aligned}
& \tau_{x x}=2 \mu \frac{\partial(\dot{x}, y)}{\partial(\alpha, \beta)}, \\
& \tau_{y y}=2 \mu \frac{\partial(x, \dot{y})}{\partial(\alpha, \beta)},
\end{aligned}
$$

and

$$
\tau_{x y}=\tau_{y x}=\mu\left[\frac{\partial(x, \dot{x})}{\partial(\alpha, \beta)}+\frac{\partial(\dot{y}, y)}{\partial(\alpha, \beta)}\right] .
$$

In the equations above, an overdot is used to denote time derivative, $p$ is the pressure, $\rho$ is the fluid density, and $\mu$ is the dynamic viscosity. Gravity is ignored.

On the two walls, the particles undergo motion in the vertical direction only

$$
\begin{aligned}
& \dot{x}=0, \quad \dot{y}=\dot{\zeta} \text { on } \beta=0, \\
& \dot{x}=0, \quad \dot{y}=\dot{\eta} \text { on } \beta=h,
\end{aligned}
$$

where $\zeta$ and $\eta$ are given in Eqs. (1) and (2), respectively.

On the basis of small-amplitude displacements, the variables may be expanded as follows [13]:

$$
(x, y, p)=(\alpha, \beta, 0)+\epsilon\left(x^{(1)}, y^{(1)}, p^{(1)}\right)+\epsilon^{2}\left(x^{(2)}, y^{(2)}, p^{(2)}\right)+\cdots .
$$

Perturbation equations are obtainable for the $O(\epsilon)$ and $O\left(\epsilon^{2}\right)$ variables on substituting Eq. (13) into the Lagrangian equations above and collecting terms of equal powers of $\epsilon$. From here on, we shall use the subscripts $\alpha$ and $\beta$ to denote the derivatives with respect to the corresponding spatial variables.

\section{FIRST-ORDER PROBLEM}

At $O(\epsilon)$, equations of motion (5)-(7) yield

$$
\begin{gathered}
x_{\alpha}^{(1)}+y_{\beta}^{(1)}=0, \\
\ddot{x}^{(1)}+\rho^{-1} p_{\alpha}^{(1)}-\nu\left(\dot{x}_{\alpha \alpha}^{(1)}+\dot{x}_{\beta \beta}^{(1)}\right)=0,
\end{gathered}
$$

and

$$
\ddot{y}^{(1)}+\rho^{-1} p_{\beta}^{(1)}-\nu\left(\dot{y}_{\alpha \alpha}^{(1)}+\dot{y}_{\beta \beta}^{(1)}\right)=0,
$$

where $\nu=\mu / \rho$ is the kinematic viscosity of the fluid. The boundary conditions are

$$
\begin{aligned}
& x^{(1)}=0, \quad y^{(1)}=\operatorname{Re}\left[a e^{i(k \alpha-\sigma t)}\right] \text { on } \beta=0, \\
& x^{(1)}=0, \quad y^{(1)}=\operatorname{Re}\left[b e^{i(k \alpha-\sigma t)}\right] \text { on } \beta=h .
\end{aligned}
$$

The oscillatory fluid motion consists of two components. The first component is induced directly by the peristaltic forcing, and hence has the same spatial and temporal periodic dependence as given by $\exp [i(k \alpha-\sigma t)]$. The second component is induced by the existence of end walls of the closed channel causing a flow that is periodic in time but uniform horizontally [6]. Solutions to the leading-order problem can be found by separating the variables into $\alpha$-periodic and $\alpha$-independent modes accordingly:

$$
\left(x^{(1)}, y^{(1)}, p^{(1)}\right)=\operatorname{Re}\left\{(\tilde{x}, \tilde{y}, \widetilde{p}) e^{i(k \alpha-\sigma t)}+(\widetilde{\widetilde{x}}, 0, \widetilde{\widetilde{p}}) e^{-i \sigma t}\right\},
$$

where $\tilde{x}, \tilde{y}, \tilde{p}$, and $\widetilde{\tilde{x}}$ are functions of $\beta$, while $\tilde{\tilde{p}}$ is a linear function of $\alpha$ such that its gradient $\widetilde{\tilde{p}}_{\alpha}$ is a constant.

\section{A. Horizontal-periodic oscillatory flow}

On substituting Eq. (19), equations of motion (14)-(16) give

$$
\begin{gathered}
\tilde{x}=i k^{-1} \tilde{y}^{\prime}, \\
i \sigma^{2} \tilde{x}+k \rho^{-1} \tilde{p}+\nu \sigma\left(-k^{2} \tilde{x}+\widetilde{x}^{\prime \prime}\right)=0, \\
-\sigma^{2} \tilde{y}+\rho^{-1} \tilde{p}^{\prime}+i \nu \sigma\left(-k^{2} \tilde{y}+\widetilde{y}^{\prime \prime}\right)=0 .
\end{gathered}
$$

These equations lead to

$$
\left(\frac{d^{2}}{d \beta^{2}}-k^{2}\right)\left(\frac{d^{2}}{d \beta^{2}}-\lambda^{2}\right) \tilde{y}=0,
$$

where

$$
\lambda^{2}=k^{2}+s^{2}, \quad s^{2}=-2 i / \delta^{2},
$$

in which

$$
\delta=\sqrt{2 \nu / \sigma}
$$

is known as the Stokes layer thickness. At this point, we assume that $\delta \sim h \sim k^{-1}$ so that the viscous effect is equally significant across the fluid layer. The case of very small Stokes layer thickness will be considered in Sec. V.

The general solution to the equations above can be written as

$$
\begin{aligned}
\tilde{x}(\beta)= & i A \cosh k \beta+i B \sinh k \beta-i C \lambda k^{-1} e^{-\lambda \beta} \\
& +i D \lambda k^{-1} e^{\lambda(\beta-h)}, \\
\tilde{y}(\beta)=A & \sinh k \beta+B \cosh k \beta+C e^{-\lambda \beta}+D e^{\lambda(\beta-h)} .
\end{aligned}
$$

On using the boundary conditions $\widetilde{x}(0)=0, \tilde{y}(0)=a, \tilde{x}(h)=0$, and $\tilde{y}(h)=b$, the constant $A-D$ can be determined as follows:

$$
\begin{gathered}
A=\lambda k^{-1}\left(C-e^{-\lambda h} D\right), \\
B=\lambda k^{-1}\left(g_{1} C+g_{2} D\right), \\
C=\left(g_{6} b-g_{4} a\right) /\left(g_{3} g_{6}-g_{4} g_{5}\right),
\end{gathered}
$$




$$
D=\left(g_{3} a-g_{5} b\right) /\left(g_{3} g_{6}-g_{4} g_{5}\right),
$$

where

$$
\begin{gathered}
g_{1}=\left(-\cosh k h+e^{-\lambda h}\right) / \sinh k h, \\
g_{2}=\left(e^{-\lambda h} \cosh k h-1\right) / \sinh k h, \\
g_{3}=\lambda k^{-1}\left(\sinh k h+g_{1} \cosh k h\right)+e^{-\lambda h}, \\
g_{4}=\lambda k^{-1}\left(-e^{-\lambda h} \sinh k h+g_{2} \cosh k h\right)+1, \\
g_{5}=\lambda k^{-1} g_{1}+1, \\
g_{6}=\lambda k^{-1} g_{2}+e^{-\lambda h} .
\end{gathered}
$$

Note that $A \sim B \sim a$, while $C \sim D \sim(k \delta) a$.

\section{B. Horizontal-uniform oscillatory flow}

On substituting Eq. (19), the $x$-momentum equation gives

$$
\left(\frac{d^{2}}{d \beta^{2}}-s^{2}\right) \widetilde{\widetilde{x}}=i(\mu \sigma)^{-1} \widetilde{\tilde{p}}_{\alpha}
$$

where $s$ is given in Eq. (24), and the pressure gradient $\widetilde{\widetilde{p}}_{\alpha}$ is a constant. This equation, with the boundary conditions $\widetilde{\widetilde{x}}(0)=0$ and $\tilde{\widetilde{x}}(h)=0$, has the solution

$$
\widetilde{\widetilde{x}}(\beta)=\left(\rho \sigma^{2}\right)^{-1} \widetilde{\tilde{p}}_{\alpha}\left[1-\frac{e^{-s \beta}+e^{s(\beta-h)}}{1+e^{-s h}}\right] .
$$

The pressure gradient $\widetilde{\tilde{p}}_{\alpha}$ can be determined using the fact that the horizontal velocity vanishes at the two lateral sidewalls of the channel. To this end, we follow Yi et al. [6] to consider the net horizontal flux across a section. The sectionmean velocity is

$$
\left\langle x^{(1)}\right\rangle=\langle\widetilde{x}\rangle e^{i(k \alpha-\sigma t)}+\langle\tilde{\tilde{x}}\rangle e^{-i \sigma t},
$$

where the angular brackets denote averaging across the channel section, $\langle f\rangle \equiv h^{-1} \int_{0}^{h} f d \beta$ for any variable $f$.

Since $\left\langle x^{(1)}\right\rangle=0$ at the two sidewalls, $\left\langle x^{(1)}\right\rangle$ must also be equal to zero on sections which are away from either sidewall by a distance equal to an integer multiple of the wavelength. To simplify analysis, we may define the origin $\alpha=0$ to be located on one of these sections, or $L_{1}=n_{1} \ell$ and $L_{2}$ $=n_{2} \ell$, where $n_{1}$ and $n_{2}=n-n_{1}$ are integers. It follows that the net flux across the section $\alpha=0$ is zero at all times, implying $\langle\widetilde{\tilde{x}}=-\langle\widetilde{x}\rangle$, by which one can obtain

$$
\rho^{-1} \tilde{\tilde{p}}_{\alpha}=\frac{i \sigma^{2}(a-b)}{k h\left[1-\frac{2\left(1-e^{-s h}\right)}{s h\left(1+e^{-s h}\right)}\right]},
$$

where Eq. (20) has been used. The section-mean velocity can also be expressed as

$$
\left\langle x^{(1)}\right\rangle=-i(k h)^{-1}(a-b)\left[e^{i(k \alpha-\sigma t)}-e^{-i \sigma t}\right],
$$

which can be compared with Eq. (7) of Selverov and Stone [5]. In the particular case $a=b$ (i.e., the two waves are in phase and have the same amplitude), this horizontally uniform flow field vanishes.

\section{SECOND-ORDER PROBLEM}

The second-order flow field considered here is for a general oscillation frequency such that the Stokes layer thickness is comparable with the channel depth, $\delta \sim h$; the steadystreaming Reynolds number is, however, small such that $a$ $\ll \delta$. The theory presented in this section shares the same basis of that by Selverov and Stone [5]. In fact, we have used our expressions, as given in Eq. (59) below, to calculate Lagrangian velocity profiles that agree very well with those presented in their Fig. 10.

The $O\left(\epsilon^{2}\right)$ governing equations are as follows:

$$
\begin{gathered}
\dot{x}_{\alpha}^{(2)}+\dot{y}_{\beta}^{(2)}=-\left(x_{\alpha}^{(1)} y_{\beta}^{(1)}\right)_{t}+\left(x_{\beta}^{(1)} y_{\alpha}^{(1)}\right)_{t}, \\
\ddot{x}^{(2)}+\rho^{-1} p_{\alpha}^{(2)}-\nu\left(\dot{x}_{\alpha \alpha}^{(2)}+\dot{x}_{\beta \beta}^{(2)}\right)=X^{(2)},
\end{gathered}
$$

and

$$
\ddot{y}^{(2)}+\rho^{-1} p_{\beta}^{(2)}-\nu\left(\dot{y}_{\alpha \alpha}^{(2)}+\dot{y}_{\beta \beta}^{(2)}\right)=Y^{(2)},
$$

where the forcing terms can be decomposed into inviscid and viscous parts: $X^{(2)}=X_{I}^{(2)}+X_{V}^{(2)}$ and $Y^{(2)}=Y_{I}^{(2)}+Y_{V}^{(2)}$, in which

$$
X_{I}^{(2)}=-\ddot{x}^{(1)} x_{\alpha}^{(1)}-\ddot{y}^{(1)} y_{\alpha}^{(1)},
$$

$$
\begin{aligned}
& X_{V}^{(2)}= \nu\left\{x_{\alpha}^{(1)}\left(-\dot{x}_{\alpha \alpha}^{(1)}+3 \dot{x}_{\beta \beta}^{(1)}\right)+y_{\alpha}^{(1)}\left(\dot{y}_{\alpha \alpha}^{(1)}+\dot{y}_{\beta \beta}^{(1)}\right)-2 \dot{x}_{\alpha \beta}^{(1)}\left(y_{\alpha}^{(1)}+x_{\beta}^{(1)}\right)\right. \\
&\left.-\dot{x}_{\alpha}^{(1)}\left(x_{\alpha \alpha}^{(1)}+x_{\beta \beta}^{(1)}\right)-\dot{x}_{\beta}^{(1)}\left(y_{\alpha \alpha}^{(1)}+y_{\beta \beta}^{(1)}\right)\right\}, \\
& Y_{I}^{(2)}=-\ddot{x}^{(1)} x_{\beta}^{(1)}-\ddot{y}^{(1)} y_{\beta}^{(1)}, \\
& Y_{V}^{(2)}= \nu\left\{x_{\beta}^{(1)}\left(\dot{x}_{\alpha \alpha}^{(1)}+\dot{x}_{\beta \beta}^{(1)}\right)+y_{\beta}^{(1)}\left(3 \dot{y}_{\alpha \alpha}^{(1)}-\dot{y}_{\beta \beta}^{(1)}\right)-2 \dot{y}_{\alpha \beta}^{(1)}\left(y_{\alpha}^{(1)}+x_{\beta}^{(1)}\right)\right. \\
&\left.-\dot{y}_{\alpha}^{(1)}\left(x_{\alpha \alpha}^{(1)}+x_{\beta \beta}^{(1)}\right)-\dot{y}_{\beta}^{(1)}\left(y_{\alpha \alpha}^{(1)}+y_{\beta \beta}^{(1)}\right)\right\} .
\end{aligned}
$$

All these terms, which consist of products of the first-order variables, are responsible for the generation of steady currents in the second order.

The steady component of the Lagrangian drift is called the mass transport velocity [12], and we may define

$$
(u, v) \equiv\left(\overline{\dot{x}}^{(2)}, \overline{\dot{y}}^{(2)}\right),
$$

where the overbar denotes time average over a period. Suppose a steady field of Lagrangian streaming is established in the entire channel. The mass transport velocity components are governed by the time-averaged Eqs. (43)-(45), which read as below:

$$
\begin{gathered}
u_{\alpha}+v_{\beta}=0, \\
\rho^{-1} \bar{p}_{\alpha}^{(2)}-\nu\left[u_{\alpha \alpha}+u_{\beta \beta}\right]=\bar{X}^{(2)},
\end{gathered}
$$

and

$$
\rho^{-1} \bar{p}_{\beta}^{(2)}-\nu\left[v_{\alpha \alpha}+v_{\beta \beta}\right]=\bar{Y}^{(2)} .
$$

The time-mean forcing terms can be separated into two parts, the first part being independent of $\alpha$, and the second part being proportional to $\exp (i k \alpha)$ : 


$$
\left(\bar{X}^{(2)}, \bar{Y}^{(2)}\right)=(\tilde{X}, \tilde{Y})+\operatorname{Re}\left\{(\tilde{\widetilde{X}}, \widetilde{\widetilde{Y}}) e^{i k \alpha}\right\},
$$

where $\tilde{X}, \tilde{Y}, \widetilde{\tilde{X}}$, and $\widetilde{\tilde{Y}}$ are functions of $\beta$ only. On substituting Eq. (19) into Eqs. (46)-(49), followed by time averaging, we get

$$
\widetilde{X}=\tilde{X}_{V}=\nu \sigma k \operatorname{Re}\left\{\frac{1}{2} k^{2}|\tilde{y}|^{2}-2 \widetilde{x}^{*} \widetilde{x}^{\prime \prime}-\widetilde{y}^{*} \tilde{y}^{\prime \prime}-\frac{3}{2}\left|\widetilde{x}^{\prime}\right|^{2}\right\},
$$

$$
\begin{aligned}
& \tilde{Y}=\tilde{Y}_{I}+\tilde{Y}_{V}=\frac{1}{2} \sigma^{2} \operatorname{Re}\left\{\widetilde{x}^{*} \widetilde{x}^{\prime}+\widetilde{y}^{*} \tilde{y}^{\prime}+\widetilde{\widetilde{x}}^{*} \widetilde{\tilde{x}}^{\prime}\right\}+\frac{1}{2} \nu \sigma \operatorname{Re}\left\{-i \widetilde{x}^{* \prime} \widetilde{x}^{\prime \prime}\right. \\
& \left.-3 i k^{2} \widetilde{x}^{*} \widetilde{x}^{\prime}-k \widetilde{y} \widetilde{x}^{* \prime \prime}-k^{3} \widetilde{x}^{*} \tilde{y}-i \tilde{\widetilde{x}^{\prime \prime}} \tilde{\widetilde{x}}^{\prime \prime}\right\}, \\
& \widetilde{\tilde{X}}=\widetilde{\tilde{X}}_{I}+\widetilde{\widetilde{X}}_{V}=\frac{1}{2} i \sigma^{2} k \tilde{x} \widetilde{x}^{*}+\nu \sigma k\left[-2 \widetilde{x} \widetilde{x}^{* \prime \prime}+\frac{1}{2} i k \tilde{y} \widetilde{x}^{* \prime}-\frac{3}{2} \widetilde{x}^{\prime} \widetilde{x}^{\prime \prime}\right],
\end{aligned}
$$

$$
\begin{aligned}
& \widetilde{\widetilde{Y}}=\tilde{\widetilde{Y}}_{I}+\widetilde{\widetilde{Y}}_{V}=\frac{1}{2} \sigma^{2}\left(\widetilde{x} \widetilde{\tilde{x}}^{*}\right)^{\prime}+\frac{1}{2} \nu \sigma\left[i \widetilde{x}^{\prime} \widetilde{x}^{* \prime \prime}+3 i k^{2} \widetilde{x} \widetilde{x}^{* \prime}\right. \\
& \left.-k \widetilde{y} \widetilde{x}^{\prime \prime \prime}-i \widetilde{x}^{\prime \prime} \widetilde{x}^{\prime \prime}\right],
\end{aligned}
$$

where an asterisk is used to denote complex conjugate. We may accordingly decompose the time-mean variables into

$$
\left(u, v, \bar{p}^{(2)}\right)=(\tilde{U}, 0, \widetilde{P})+\operatorname{Re}\left\{(\widetilde{\widetilde{U}}, \widetilde{\widetilde{V}}, \widetilde{\widetilde{P}}) e^{i k \alpha}\right\},
$$

where $\widetilde{U}, \widetilde{\widetilde{U}}, \widetilde{\widetilde{V}}$, and $\widetilde{\widetilde{P}}$ are pure functions of $\beta$, while $\widetilde{P}$ is a linear function of $\alpha$ such that $\widetilde{P}_{\alpha}$ is a constant. Obviously, the first and the second parts on the right-hand side of Eq. (59) represent components of the steady flow field which are horizontally uniform (one dimensional with parallel streamlines) and horizontally periodic (two dimensional with cellular recirculating streamlines), respectively.

\section{A. One-dimensional steady streaming}

We may infer from Eq. (53) that the mean pressure gradient $\widetilde{P}_{\alpha}$ does not depend on $\beta$, and it is in fact a constant pressure gradient responsible for the generation of a return current in a closed domain [14]. Hence, the flow is governed by, from Eq. (52),

$$
\nu \widetilde{U}^{\prime \prime}=-\tilde{X}+\rho^{-1} \widetilde{P}_{\alpha},
$$

which can be solved by integration twice with respect to $\beta$. The two integration constants and the pressure gradient $\widetilde{P}_{\alpha}$ are determined using the boundary conditions $\tilde{U}(0)=\tilde{U}(h)$ $=0$, and the zero-flux condition $\langle\widetilde{U}\rangle=0$. After some algebra, we get

$$
\begin{aligned}
\tilde{U}(\beta)= & E(\beta)-E_{0}+6\left[\left(\langle E\rangle-\frac{1}{2} E_{h}-\frac{1}{2} E_{0}\right)(\beta / h)^{2}\right. \\
& \left.-\left(\langle E\rangle-\frac{1}{3} E_{h}-\frac{2}{3} E_{0}\right)(\beta / h)\right],
\end{aligned}
$$

$$
\rho^{-1} \widetilde{P}_{\alpha}=12 \nu h^{-2}\left(\langle E\rangle-\frac{1}{2} E_{h}-\frac{1}{2} E_{0}\right),
$$

where $E_{0}=E(0), E_{h}=E(h)$,

$$
\begin{aligned}
E(\beta)= & \sigma k \operatorname{Re}\left\{\frac{1}{2}\left(|A|^{2}+|B|^{2}\right) \cosh 2 k \beta+A^{*} B \sinh 2 k \beta\right. \\
& +\frac{1}{8} \lambda_{r}^{-2} k^{2}\left(1+3|\lambda|^{2} k^{-2}\right)\left(1+|\lambda|^{2} k^{-2}\right)\left(|C|^{2} e^{-2 \lambda_{r} \beta}\right. \\
& \left.+|D|^{2} e^{2 \lambda_{r}(\beta-h)}\right)-2 \lambda k^{-1}\left(C e^{-\lambda \beta}-D e^{\lambda(\beta-h)}\right) \\
& \times\left(A^{*} \cosh k \beta+B^{*} \sinh k \beta\right)-\frac{1}{4} \lambda_{i}^{-2} k^{2}\left(1-3|\lambda|^{2} k^{-2}\right) \\
& \left.\times\left(1-|\lambda|^{2} k^{-2}\right) C^{*} D e^{\left(2 i \lambda_{i} \beta-\lambda h\right)}\right\},
\end{aligned}
$$

and

$$
\begin{aligned}
\langle E\rangle= & \sigma k \operatorname{Re}\left\{\frac{1}{4}(k h)^{-1} \sinh 2 h k\left(|A|^{2}+|B|^{2}\right)\right. \\
& +\frac{1}{2}(k h)^{-1}(\cosh 2 k h-1) A^{*} B+\frac{1}{16} \lambda_{r}^{-3} k^{2} h^{-1}(1 \\
& \left.+3|\lambda|^{2} k^{-2}\right)\left(1+|\lambda|^{2} k^{-2}\right)\left(1-e^{-2 \lambda} r_{r} h\right)\left(|C|^{2}+|D|^{2}\right) \\
& -\lambda k^{-1}(\lambda-k)^{-1} h^{-1}\left(1-e^{-(\lambda-k) h}\right)\left(A^{*}+B^{*}\right) C \\
& -\lambda k^{-1}(\lambda+k)^{-1} h^{-1}\left(1-e^{-(\lambda+k) h}\right)\left(A^{*}-B^{*}\right) C \\
& -\lambda k^{-1}(\lambda-k)^{-1} h^{-1}\left(e^{-\lambda h}-e^{-k h}\right)\left(A^{*}-B^{*}\right) D-\lambda k^{-1}(\lambda \\
& +k)^{-1} h^{-1}\left(e^{-\lambda h}-e^{k h}\right)\left(A^{*}+B^{*}\right) D-\frac{1}{8} i \lambda_{i}^{-3} k^{2} h^{-1}(1 \\
& \left.\left.-3|\lambda|^{2} k^{-2}\right)\left(1-|\lambda|^{2} k^{-2}\right) e^{-\lambda h}\left(1-e^{2 i \lambda_{i} h}\right) C^{*} D\right\},
\end{aligned}
$$

in which $\lambda_{r}$ and $\lambda_{i}$ are the real and imaginary parts of $\lambda$, respectively. One should find that the inviscid forcings $X_{I}^{(2)}$ and $Y_{I}^{(2)}$ do not contribute to this Lagrangian transport velocity field.

\section{B. Cellular steady streaming}

Equations (51)-(53) give

$$
\widetilde{\widetilde{U}}=i k^{-1} \widetilde{\widetilde{V}}^{\prime},
$$

$$
\begin{aligned}
& \rho^{-1} \tilde{\widetilde{P}}=-i k^{-1} \tilde{\widetilde{X}}+\nu\left(-\tilde{\widetilde{V}}^{\prime}+k^{-2} \tilde{\widetilde{V}}^{\prime \prime \prime}\right), \\
& \left(\frac{d^{2}}{d \beta^{2}}-k^{2}\right)^{2} \tilde{\widetilde{V}}=\nu^{-1} k^{2}\left(\widetilde{\widetilde{Y}}+i k^{-1} \tilde{\widetilde{X}}^{\prime}\right) .
\end{aligned}
$$

The general solution is

$$
\begin{aligned}
\widetilde{\widetilde{U}}(\beta)= & i F \cosh k \beta+i G k^{-1} \sinh k \beta+i G \beta \cosh k \beta \\
& +i H \sinh k \beta+i I k^{-1} \cosh k \beta+i I \beta \sinh k \beta \\
& +i k^{-1} \tilde{\widetilde{V}}_{p}^{\prime}(\beta),
\end{aligned}
$$




$$
\begin{aligned}
\widetilde{\widetilde{V}}(\beta)= & F \sinh k \beta+G \beta \sinh k \beta+H \cosh k \beta \\
& +I \beta \cosh k \beta+\widetilde{\widetilde{V}}_{p}(\beta) .
\end{aligned}
$$

The particular solution is

$$
\begin{aligned}
\widetilde{\tilde{V}}_{p}(\beta)= & \gamma_{1} A \sinh \left[\left(s^{*}+k\right) \beta-s^{*} h / 2\right]+\gamma_{2} A \sinh \left[\left(s^{*}-k\right) \beta\right. \\
& \left.-s^{*} h / 2\right]+\gamma_{1} B \cosh \left[\left(s^{*}+k\right) \beta-s^{*} h / 2\right] \\
& -\gamma_{2} B \cosh \left[\left(s^{*}-k\right) \beta-s^{*} h / 2\right]+\gamma_{3} C e^{-\lambda \beta} \cosh \left[s^{*}(\beta\right. \\
& -h / 2)]+\gamma_{3} D e^{\lambda(\beta-h)} \cosh \left[s^{*}(\beta-h / 2)\right],
\end{aligned}
$$

where

$$
\begin{aligned}
\left(\gamma_{1}, \gamma_{2}, \gamma_{3}\right)= & -\frac{k^{2} s^{* 2} \widetilde{\tilde{p}}_{\alpha}^{*}}{\rho \sigma \cosh \left(s^{*} h / 2\right)}\left[\frac{s^{*}+k}{\left(s^{* 2}+2 s^{*} k\right)^{2}},\right. \\
& \left.\frac{s^{*}-k}{\left(s^{* 2}-2 s^{*} k\right)^{2}}, \frac{k\left(3 \lambda^{2} k^{-2}-1\right)}{\left(2 s^{*} \lambda\right)^{2}}\right] .
\end{aligned}
$$

Using the boundary conditions $\widetilde{\widetilde{U}}(0)=0, \tilde{\widetilde{U}}(h)=0, \tilde{\widetilde{V}}(0)=0$, and $\widetilde{\widetilde{V}}(h)=0$, the constants $F-I$ can be determined as follows:

$$
\begin{gathered}
F=-k^{-1}\left[I+\widetilde{\widetilde{V}}_{p}^{\prime}(0)\right], \\
H=-\widetilde{\widetilde{V}}_{p}(0), \\
G=\left(g_{7} r_{1}-g_{8} r_{2}\right) /\left[\sinh ^{2} k h-(k h)^{2}\right], \\
I=\left(g_{7} r_{2}-g_{9} r_{1}\right) /\left[\sinh ^{2} k h-(k h)^{2}\right],
\end{gathered}
$$

where

$$
\begin{gathered}
g_{7}=h k \sinh h k, \quad g_{8}=-\sinh k h+k h \cosh k h, \\
g_{9}=\sinh k h+k h \cosh k h, \\
r_{1}=\widetilde{\widetilde{V}}_{p}^{\prime}(0) \sinh k h+k \widetilde{\widetilde{V}}_{p}(0) \cosh k h-k \widetilde{\widetilde{V}}_{p}(h), \\
r_{2}=\widetilde{\widetilde{V}}_{p}^{\prime}(0) \cosh k h+k \widetilde{\widetilde{V}}_{p}(0) \sinh k h-\widetilde{\widetilde{V}}_{p}^{\prime}(h) .
\end{gathered}
$$

Again, one may check that the inviscid forcings $\widetilde{\widetilde{X}}_{I}$ and $\widetilde{\tilde{Y}}_{I}$ give a zero the right-hand side of Eq. (67), and hence do not contribute to this transport velocity field as well. Also, this cellular steady streaming vanishes when $a=b$.

\section{FAST OSCILLATION}

Let us examine in this section the case when the oscillation frequency is so high that the Stokes layer thickness becomes comparable with the wave amplitude: $\delta \sim a \ll h, \ell$, or the steady-streaming Reynolds number $\operatorname{Re}_{s}=O(1)$. Under this condition, the flow domain can be divided into three regions: top boundary layer, core, and bottom boundary layer. The viscous flow is now confined largely to the top and bottom Stokes boundary layers, which are much thinner than the channel depth. The sharp contrast in length scales is now extended to $|\lambda| \sim|s| \sim \delta^{-1} \gg k$ or $|\lambda h| \sim|s h| \sim \epsilon^{-1} \gg 1$. Hence, terms such as $\exp (-\lambda h)$ and $\exp (-s h)$ can be dropped from the solutions. As a result of change in the orders of terms, the analysis, especially for the two-dimensional steady streaming, needs to be modified.

\section{A. First-order solution}

Recall that the first-order solution is expressible by Eq. (19), where the horizontal-periodic part of the solution is given in Eqs. (26) and (27), while the horizontal-independent part of the solution is given in Eqs. (39) and (41). In the expressions for $\tilde{x}$ and $\tilde{y}$, the terms associated with the functions $\sinh k \beta$ and $\cosh k \beta$ correspond to the nonviscous part of the solution and are important throughout the fluid column. The terms associated with the functions $\exp (-\lambda \beta)$ and $\exp [\lambda(\beta-h)]$ are significant only in the bottom and the top boundary layers, respectively. Likewise, in the expression for $\tilde{\tilde{x}}$, the terms associated with $\exp (-s \beta)$ and $\exp [s(\beta-h)]$ are appreciable only in the bottom and the top boundary layers, respectively.

The solution for flow in the inviscid core can be summarized as follows, where the quantities are distinguished by subscript $I$. For the horizontal-periodic part,

$$
\begin{gathered}
\tilde{x}_{I}(\beta)=i A \cosh k \beta+i B \sinh k \beta, \\
\tilde{y}_{I}(\beta)=A \sinh k \beta+B \cosh k \beta,
\end{gathered}
$$

where $A=(b-a \cosh k h) / \sinh k h$ and $B=a$. For the horizontal-independent part,

$$
\widetilde{\widetilde{x}}_{I}=\left(\rho \sigma^{2}\right)^{-1} \widetilde{\widetilde{p}}_{I \alpha}=i(k h)^{-1}(a-b) .
$$

\section{B. Second-order solution}

\section{Boundary layers}

Applying the standard boundary layer approximations, namely, $\partial / \partial \beta \gg \partial / \partial \alpha$ and so on, Eqs. (52) and (53) can be simplified to yield

$$
\nu \frac{\partial^{2} u_{b l}}{\partial \beta^{2}}=\frac{\partial}{\partial \alpha} \int \bar{Y}_{b l}^{(2)} d \beta-\bar{X}_{b l}^{(2)},
$$

where the subscript $b l$ is used to denote a boundary-layer quantity. With the separation into components by Eqs. (54) and (59), the governing equations are

$$
\nu \tilde{U}_{b l}^{\prime \prime}=-\tilde{X}_{b l}
$$

for the one-dimensional steady streaming and

$$
\nu \widetilde{\widetilde{U}}_{b l}^{\prime \prime}=i k \int \widetilde{\widetilde{Y}}_{b l} d \beta-\tilde{\widetilde{X}}_{b l}, \quad \widetilde{\widetilde{V}}_{b l}=-i k \int \widetilde{\tilde{U}}_{b l} d \beta
$$

for the cellular steady streaming. Source terms that are dominant in the boundary layers can be identified from Eqs. (55), (57), and (58) as

$$
\tilde{X}_{b l}=\nu \sigma k \operatorname{Re}\left\{-2 \tilde{x}^{*} \tilde{x}^{\prime \prime}-\frac{3}{2}\left|\tilde{x}^{\prime}\right|^{2}\right\},
$$




$$
\begin{aligned}
& \tilde{\tilde{X}}_{b l}=\frac{1}{2} i \sigma^{2} k \widetilde{x} \widetilde{\tilde{x}}^{*}+\nu \sigma k\left[-2 \widetilde{x} \widetilde{\tilde{x}}^{* \prime \prime}-\frac{3}{2} \widetilde{x}^{\prime} \widetilde{\tilde{x}}^{* \prime}\right], \\
& \tilde{\widetilde{Y}}_{b l}=\frac{1}{2} \sigma^{2}\left(\widetilde{x} \widetilde{\tilde{x}}^{*}\right)^{\prime}+\frac{1}{2} \nu \sigma\left[i \widetilde{x}^{\prime} \widetilde{\tilde{x}}^{* \prime \prime}-i \widetilde{x}^{\prime \prime} \widetilde{\tilde{x}}^{* \prime}\right] .
\end{aligned}
$$

One also finds that the forcing in Eq. (84) can be written as

$$
i k \int \widetilde{\widetilde{Y}}_{b l} d \beta-\widetilde{\tilde{X}}_{b l}=\nu \sigma k\left[-\int \tilde{x}^{\prime} \widetilde{\widetilde{x}}^{* \prime \prime} d \beta+2\left(\widetilde{x} \widetilde{\tilde{x}}^{* \prime}\right)\right] .
$$

Let us use in the following expressions subscripts $b b l$ and $t b l$ to denote, respectively, quantities of the bottom and the top boundary layers. On performing integration across either boundary layer, the functions $\sinh k \beta$ and $\cosh k \beta$ are slow varying and can be regarded as constants.

We get from Eqs. (83) and (85) the one-dimensional mass transport velocity in the bottom boundary layer:

$$
\begin{aligned}
\tilde{U}_{b b l}(\beta)= & -\sigma k \operatorname{Re}\left\{2 \lambda k^{-1} C\left(A^{*} \cosh k \beta+B^{*} \sinh k \beta\right) e^{-\lambda \beta}\right. \\
& \left.-\frac{3}{8}|C|^{2} \mid \lambda^{2}{ }^{2} k^{-2} \lambda_{r}^{-2} e^{-2 \lambda_{r} \beta}\right\}+\widetilde{U}_{b b l}^{\infty}
\end{aligned}
$$

and that in the top boundary layer:

$$
\begin{aligned}
\tilde{U}_{t b l}(\beta)= & -\sigma k \operatorname{Re}\left\{-2 \lambda k^{-1} D\left(A^{*} \cosh k \beta\right.\right. \\
& \left.+B^{*} \sinh k \beta\right) e^{\lambda(\beta-h)} \\
& \left.-\frac{3}{8}|D|^{2}\left|\lambda^{2}\right|^{2} k^{-2} \lambda_{r}^{-2} e^{2 \lambda_{r}(\beta-h)}\right\}+\tilde{U}_{t b l}^{\infty},
\end{aligned}
$$

where

$$
\begin{aligned}
\tilde{U}_{b b l}^{\infty} & =\sigma k \operatorname{Re}\left\{2 \lambda k^{-1} C A^{*}-\frac{3}{8}|C|^{2}\left|\lambda^{2}\right|^{2} k^{-2} \lambda_{r}^{-2}\right\}, \\
\tilde{U}_{t b l}^{\infty} & =\sigma k \operatorname{Re}\left\{-2 \lambda k^{-1} D\left(A^{*} \cosh k h+B^{*} \sinh k h\right)\right. \\
& \left.-\frac{3}{8}|D|^{2}\left|\lambda^{2}\right|^{2} k^{-2} \lambda_{r}^{-2}\right\}
\end{aligned}
$$

are the Lagrangian drifts at the outer edge of the respective boundary layers. The boundary conditions $\tilde{U}_{b b l}(0)=\tilde{U}_{b b l}^{\prime}(\infty)$ $=0$ and $\tilde{U}_{t b l}(h)=\tilde{U}_{t b l}^{\prime}(-\infty)=0$ have been used. Here, infinity means a sufficiently large distance from the boundary layer.

For the cellular steady streaming, we get from Eqs. (84) and (88) its mass transport velocity components in the bottom boundary layer:

$$
\begin{aligned}
\widetilde{\tilde{U}}_{b b l}(\beta)= & i(\rho \sigma)^{-1} k \widetilde{\tilde{p}}_{I \alpha}^{*}\left[-2(A \cosh k \beta+B \sinh k \beta) e^{-\lambda^{*} \beta}\right. \\
& \left.+\frac{3}{4} C|\lambda|^{2} k^{-1} \lambda_{r}^{-1} e^{-2 \lambda_{r} \beta}\right]+\widetilde{\tilde{U}}_{b b l}^{\infty}
\end{aligned}
$$

$$
\begin{aligned}
\widetilde{\widetilde{V}}_{b b l}(\beta)= & (\rho \sigma)^{-1} k^{2} \widetilde{\tilde{p}}_{I \alpha}^{*}\left[2\left(\lambda^{*}\right)^{-1}(A \cosh k \beta+B \sinh k \beta) e^{-\lambda^{*} \beta}\right. \\
& \left.-\frac{3}{8} C|\lambda|^{2} k^{-1} \lambda_{r}^{-2} e^{-2 \lambda_{r} \beta}\right]-i k \widetilde{\widetilde{U}}_{b b l}^{\infty} \beta+\widetilde{\widetilde{V}}_{b b l}^{\infty}
\end{aligned}
$$

and those in the top boundary layer:

$$
\begin{aligned}
\tilde{\widetilde{U}}_{t b l}(\beta)= & i(\rho \sigma)^{-1} k \widetilde{\tilde{p}}_{I \alpha}^{*}\left[-2(A \cosh k \beta+B \sinh k \beta) e^{\lambda^{*}(\beta-h)}\right. \\
& \left.-\frac{3}{4} D|\lambda|^{2} k^{-1} \lambda_{r}^{-1} e^{2 \lambda_{r}(\beta-h)}\right]+\widetilde{\widetilde{U}}_{t b l}^{\infty}, \\
\widetilde{\widetilde{V}}_{t b l}(\beta)= & (\rho \sigma)^{-1} k^{2} \widetilde{\tilde{p}}_{I \alpha}^{*}\left[-2\left(\lambda^{*}\right)^{-1}(A \cosh k \beta\right. \\
& \left.+B \sinh k \beta) e^{\lambda^{*}(\beta-h)}-\frac{3}{8} D|\lambda|^{2} k^{-1} \lambda_{r}^{-2} e^{2 \lambda_{r}(\beta-h)}\right] \\
& -i k \widetilde{\tilde{U}}_{t b l}^{\infty}(\beta-h)+\widetilde{\widetilde{V}}_{t b l}^{\infty}
\end{aligned}
$$

where

$$
\begin{gathered}
\widetilde{\tilde{U}}_{b b l}^{\infty}=i(\rho \sigma)^{-1} k \widetilde{\tilde{p}}_{I \alpha}^{*}\left[2 A-\frac{3}{4} C|\lambda|^{2} k^{-1} \lambda_{r}^{-1}\right] \\
\widetilde{\widetilde{V}}_{b b l}^{\infty}=(\rho \sigma)^{-1} k^{2} \widetilde{\tilde{p}}_{I \alpha}^{*}\left[-2\left(\lambda^{*}\right)^{-1} A+\frac{3}{8} C|\lambda|^{2} k^{-1} \lambda_{r}^{-2}\right] \\
\widetilde{\tilde{U}}_{t b l}^{\infty}=i(\rho \sigma)^{-1} k \widetilde{\tilde{p}}_{I \alpha}^{*}[2(A \cosh k h+B \sinh k h) \\
\left.+\frac{3}{4} D|\lambda|^{2} k^{-1} \lambda_{r}^{-1}\right] \\
{\widetilde{\tilde{V}_{t b l}}}^{\infty}=(\rho \sigma)^{-1} k^{2} \widetilde{\tilde{p}}_{I \alpha}^{*}\left[2\left(\lambda^{*}\right)^{-1}(A \cosh k h+B \sinh k h)\right. \\
\left.+\frac{3}{8} D|\lambda|^{2} k^{-1} \lambda_{r}^{-2}\right]
\end{gathered}
$$

are the components of the Lagrangian drifts at the outer edge of the respective boundary layers. The conditions of zero velocity at the wall and zero velocity gradient far from the boundary layer are also applied.

\section{Core region}

The viscous effect becomes subdominant in the core region. One can check that the viscous stress and source terms in Eqs. (44) and (45) are now two orders of magnitude smaller than other terms in the equations. Hence, the secondorder equations contain only the inviscid terms:

$$
\begin{aligned}
& \ddot{x}^{(2)}+\rho^{-1} p_{\alpha}^{(2)}=X_{I}^{(2)}, \\
& \ddot{y}^{(2)}+\rho^{-1} p_{\beta}^{(2)}=Y_{I}^{(2)},
\end{aligned}
$$

where $X_{I}^{(2)}$ and $Y_{I}^{(2)}$ are given in Eqs. (46) and (48).

The third-order equations are also without the viscous terms: 


$$
\begin{aligned}
x_{\alpha}^{(3)}+y_{\beta}^{(3)}= & -x_{\alpha}^{(1)} y_{\beta}^{(2)}-x_{\alpha}^{(2)} y_{\beta}^{(1)}+x_{\beta}^{(1)} y_{\alpha}^{(2)}+x_{\beta}^{(2)} y_{\alpha}^{(1)}, \\
\ddot{x}^{(3)}+\rho^{-1} p_{\alpha}^{(3)}= & \ddot{x}^{(1)} y_{\beta}^{(2)}-\ddot{y}^{(1)} y_{\alpha}^{(2)}+\left[\ddot{x}^{(2)}+\ddot{x}^{(1)} x_{\alpha}^{(1)}\right] y_{\beta}^{(1)} \\
& -\left[\ddot{y}^{(2)}+\ddot{x}^{(1)} x_{\beta}^{(1)}\right] y_{\alpha}^{(1)}, \\
\ddot{y}^{(3)}+\rho^{-1} p_{\beta}^{(3)}= & \ddot{y}^{(1)} x_{\alpha}^{(2)}-\ddot{x}^{(1)} x_{\beta}^{(2)}+\left[\ddot{y}^{(2)}+\ddot{y}^{(1)} y_{\beta}^{(1)}\right] x_{\alpha}^{(1)} \\
& -\left[\ddot{x}^{(2)}+\ddot{y}^{(1)} y_{\alpha}^{(1)}\right] x_{\beta}^{(1)} .
\end{aligned}
$$

The steady streaming can then be found from the fourthorder momentum equations in which the second-order viscous terms show up:

$$
\begin{aligned}
\ddot{x}^{(4)}+\rho^{-1} p_{\alpha}^{(4)}-\nu\left(\dot{x}_{\alpha \alpha}^{(2)}+\dot{x}_{\beta \beta}^{(2)}\right)= & X_{V}^{(2)}-\rho^{-1}\left(p_{\alpha}^{(2)} y_{\beta}^{(2)}+p_{\alpha}^{(1)} y_{\beta}^{(3)}\right. \\
& +p_{\alpha}^{(3)} y_{\beta}^{(1)}-p_{\beta}^{(2)} y_{\alpha}^{(2)}-p_{\beta}^{(1)} y_{\alpha}^{(3)} \\
& \left.-p_{\beta}^{(3)} y_{\alpha}^{(1)}\right), \\
\ddot{y}^{(4)}+\rho^{-1} p_{\beta}^{(4)}-\nu\left(\dot{y}_{\alpha \alpha}^{(2)}+\dot{y}_{\beta \beta}^{(2)}\right)= & Y_{V}^{(2)}-\rho^{-1}\left(x_{\alpha}^{(2)} p_{\beta}^{(2)}+x_{\alpha}^{(1)} p_{\beta}^{(3)}\right. \\
& +x_{\alpha}^{(3)} p_{\beta}^{(1)}-x_{\beta}^{(2)} p_{\alpha}^{(2)}-x_{\beta}^{(1)} p_{\alpha}^{(3)} \\
& \left.-x_{\beta}^{(3)} p_{\alpha}^{(1)}\right),
\end{aligned}
$$

where $X_{V}^{(2)}$ and $Y_{V}^{(2)}$ are given in Eqs. (47) and (49). Upon taking time average of these equations, we get

$$
\begin{aligned}
\rho^{-1} \bar{p}_{\alpha}^{(4)}-\nu\left(u_{\alpha \alpha}^{(2)}+u_{\beta \beta}^{(2)}\right)= & \bar{X}_{V}^{(2)}-\rho^{-1}\left(\overline{p_{\alpha}^{(2)} y_{\beta}^{(2)}}+\overline{p_{\alpha}^{(1)} y_{\beta}^{(3)}}+\overline{p_{\alpha}^{(3)} y_{\beta}^{(1)}}\right. \\
& \left.-\overline{p_{\beta}^{(2)} y_{\alpha}^{(2)}}-\overline{p_{\beta}^{(1)} y_{\alpha}^{(3)}}-\overline{p_{\beta}^{(3)} y_{\alpha}^{(1)}}\right), \quad(108) \\
\rho^{-1} \bar{p}_{\beta}^{(4)}-\nu\left(v_{\alpha \alpha}^{(2)}+v_{\beta \beta}^{(2)}\right)= & \bar{Y}_{V}^{(2)}-\rho^{-1}\left(\overline{x_{\alpha}^{(2)} p_{\beta}^{(2)}}+\overline{x_{\alpha}^{(1)} p_{\beta}^{(3)}}+\overline{x_{\alpha}^{(3)} p_{\beta}^{(1)}}\right. \\
& \left.-\overline{x_{\beta}^{(2)} p_{\alpha}^{(2)}}-\overline{x_{\beta}^{(1)} p_{\alpha}^{(3)}}-\overline{x_{\beta}^{(3)} p_{\alpha}^{(1)}}\right) .
\end{aligned}
$$

When compared with the previous counterparts, Eqs. (52) and (53), these equations for the steady Lagrangian drifts contain additional forcing terms that are products of secondorder variables, as well as the products of first- and thirdorder variables.

The second-order variables can be found by solving Eqs. (43), (101), and (102), where their right-hand sides are computed using the first-order inviscid solution Eqs. (79)-(81). The time-oscillatory parts of the second-order solution can be obtained as follows:

$$
\begin{aligned}
\left(x^{(2)}, y^{(2)}, p^{(2)}\right)= & \operatorname{Re}\left\{\left(x^{(21)}, 0, p^{(21)}\right) e^{2 i(k \alpha-\sigma t)}\right. \\
& \left.+\left(0,0, p^{(22)}\right) e^{i(k \alpha-2 \sigma t)}+\left(x^{(23)}, 0, p^{(23)}\right) e^{-2 i \sigma t}\right\},
\end{aligned}
$$

where

$$
\begin{gathered}
x^{(21)}=\frac{1}{4}\left(-\tilde{x}_{I} \tilde{y}_{I}^{\prime}+\tilde{x}_{I}^{\prime} \tilde{y}_{I}\right)=\frac{1}{4} i k\left(B^{2}-A^{2}\right), \\
\rho^{-1} p^{(21)}=\frac{1}{4} \sigma^{2}\left(\widetilde{x}_{I}^{2}+\tilde{y}_{I}^{2}\right)-2 i \sigma^{2} k^{-1} x^{(21)}=\frac{3}{4} \sigma^{2}\left(B^{2}-A^{2}\right),
\end{gathered}
$$

$$
\begin{aligned}
\rho^{-1} p^{(22)}= & \frac{1}{2} \sigma^{2} \tilde{x}_{I} \widetilde{\widetilde{x}}_{I}=-\frac{1}{2} \sigma^{2}(k h)^{-1}(a-b)(A \cosh k \beta \\
& +B \sinh k \beta), \\
x^{(23)}=-x^{(21)}= & \frac{1}{4} i k\left(A^{2}-B^{2}\right), \quad \rho^{-1} p_{\alpha}^{(23)}=i \sigma^{2} k\left(A^{2}-B^{2}\right) .
\end{aligned}
$$

The third-order variables are obtained likewise by solving Eqs. (103)-(105). Only the first-harmonic components may give rise to a nonzero time mean when interacting with the first-order variables. These components are of the following modes:

$$
\begin{aligned}
\left(x^{(3)}, y^{(3)}, p^{(3)}\right)= & \operatorname{Re}\left\{\left(x^{(31)}, y^{(31)}, p^{(31)}\right) e^{i(k \alpha-\sigma t)}\right. \\
& +\left(0,0, p^{(32)}\right) e^{-i(k \alpha+\sigma t)}+\left(0,0, p^{(33)}\right) e^{i(2 k \alpha-\sigma t)} \\
& \left.+\left(x^{(34)}, 0, p^{(34)}\right) e^{-i \sigma t}+\mathrm{hht}\right\},
\end{aligned}
$$

where hht stands for higher-harmonic terms, $x^{(31)}, y^{(31)}, p^{(31)}$, $p^{(32)}, p^{(33)}$, and $x^{(34)}$ are functions of $\beta$, while $p_{\alpha}^{(34)}$ is a constant.

For the $\exp [i(k \alpha-\sigma t)]$ mode, the governing equations lead to

$$
\begin{aligned}
y^{(31) \prime \prime}-k^{2} y^{(31)}= & -6 i k x^{(21)} \tilde{y}_{I}^{* \prime \prime}-\frac{1}{2} k^{2}\left(\tilde{y}_{I}^{*} \tilde{y}_{I}^{\prime 2}+2 \tilde{y}_{I} \tilde{y}_{I}^{\prime} \tilde{y}_{I}^{* \prime}\right. \\
& \left.+\tilde{y}_{I} \widetilde{y}_{I}^{*} \widetilde{y}_{I}^{\prime \prime}\right) .
\end{aligned}
$$

The solution satisfying the boundary conditions $y^{(31)}(h)$ $=y^{(31)}(0)=0$ is

$$
y^{(31)}(\beta)=J \sinh k \beta+K \cosh k \beta+y_{p}^{(31)}(\beta),
$$

where

$$
J=\left[y_{p}^{(31)}(0) \cosh k h-y_{p}^{(31)}(h)\right] / \sinh k h, \quad K=-y_{p}^{(31)}(0),
$$

and the particular solution

$$
\begin{aligned}
y_{p}^{(31)}(\beta)= & \gamma_{4} k \beta \sinh k \beta+\gamma_{5} k \beta \cosh k \beta \\
& +\gamma_{6} \sinh 3 k \beta+\gamma_{7} \cosh 3 k \beta,
\end{aligned}
$$

in which

$$
\left(\gamma_{4}, \gamma_{5}\right)=\frac{3}{4} k^{2}\left(B^{2}-A^{2}\right)\left(B^{*}, A^{*}\right)
$$

$$
\begin{aligned}
& \gamma_{6}=-\frac{1}{16} k^{2}\left(A^{*} B^{2}+A^{2} A^{*}+2 A B B^{*}\right), \\
& \gamma_{7}=-\frac{1}{16} k^{2}\left(A^{2} B^{*}+B^{2} B^{*}+2 A A^{*} B\right) .
\end{aligned}
$$

The horizontal displacement and pressure are then given by

$$
x^{(31)}=i k^{-1} y^{(31) \prime}-x^{(21)} \tilde{y}_{I}^{* \prime},
$$




$$
\begin{aligned}
\rho^{-1} p^{(31)}= & \sigma^{2} k^{-1}\left[k^{-1} y^{(31) \prime}+3 i x^{(21)} \widetilde{y}_{I}^{* \prime}+\frac{1}{4} k\left(-\widetilde{x}_{I}^{2} \widetilde{y}_{I}^{* \prime}-\widetilde{x}_{I} \widetilde{x}_{I}^{\prime} \widetilde{y}_{I}^{*}\right.\right. \\
& \left.\left.+\widetilde{x}_{I} \widetilde{x}_{I}^{* \prime} \widetilde{y}_{I}+\widetilde{x}_{I}^{*} \widetilde{x}_{I}^{\prime} \widetilde{y}_{I}\right)\right] .
\end{aligned}
$$

For the $\exp [-i(k \alpha+\sigma t)]$ and $\exp [i(2 k \alpha-\sigma t)]$ modes, the displacements are all zero, and the pressures are

$$
\begin{gathered}
\rho^{-1} p^{(32)}=-2 i \sigma^{2} k^{-1} x^{(23)} \tilde{y}_{I}^{* \prime}, \\
\rho^{-1} p^{(33)}=\frac{1}{8} \sigma^{2} \widetilde{\widetilde{x}}_{I}^{*}\left(-\widetilde{x}_{I} \widetilde{y}_{I}^{\prime}+\widetilde{x}_{I}^{\prime} \tilde{y}_{I}\right) .
\end{gathered}
$$

For the $\exp (-i \sigma t)$ mode, the vertical displacement is zero, and

$$
x^{(34)}=\left(\sigma^{2} \rho\right)^{-1} p_{\alpha}^{(34)}-\frac{1}{4} i k \tilde{\widetilde{x}}_{I}\left(\widetilde{x}_{I}^{*} \widetilde{y}_{I}-\widetilde{x}_{I} \widetilde{y}_{I}^{*}\right)^{\prime},
$$

where the pressure gradient $p_{\alpha}^{(34)}$ is determined from $\left\langle x^{(34)}\right\rangle$ $=-\left\langle x^{(31)}\right\rangle$.

We may now evaluate the forcing terms on the right-hand sides of Eqs. (108) and (109) for the steady streaming. With $\overline{p_{\alpha}^{(2)} y_{\beta}^{(2)}}=\overline{p_{\beta}^{(2)} y_{\alpha}^{(2)}}=\overline{x_{\beta}^{(2)} p_{\alpha}^{(2)}}=0$, the forcing terms consist of two groups corresponding to second-order viscous and fourthorder inviscid interaction terms

$$
\begin{aligned}
\bar{X}_{V}^{(2)}- & \left.\rho^{-1} \overline{p_{\alpha}^{(1)} y_{\beta}^{(3)}}+\overline{p_{\alpha}^{(3)} y_{\beta}^{(1)}}-\overline{p_{\beta}^{(1)} y_{\alpha}^{(3)}}-\overline{p_{\beta}^{(3)} y_{\alpha}^{(1)}}\right)=\bar{X}_{V}^{(2)}+\bar{X}_{I}^{(4)}, \\
\bar{Y}_{V}^{(2)} & -\rho^{-1}\left(\overline{x_{\alpha}^{(2)} p_{\beta}^{(2)}}+\overline{x_{\alpha}^{(1)} p_{\beta}^{(3)}}+\overline{x_{\alpha}^{(3)} p_{\beta}^{(1)}}-\overline{x_{\beta}^{(1)} p_{\alpha}^{(3)}}-\overline{x_{\beta}^{(3)} p_{\alpha}^{(1)}}\right) \\
& =\bar{Y}_{V}^{(2)}+\bar{Y}_{I}^{(4)}
\end{aligned}
$$

which can be further expressed as

$$
\begin{aligned}
\left(\bar{X}_{V}^{(2)}+\bar{X}_{I}^{(4)}, \bar{Y}_{V}^{(2)}+\bar{Y}_{I}^{(4)}\right)= & \left(\tilde{X}_{V c}+\tilde{X}_{I}^{(4)}, \tilde{Y}_{V c}+\tilde{Y}_{I}^{(4)}\right) \\
& +\operatorname{Re}\left[\left(\tilde{\widetilde{X}}_{V c}+\tilde{\widetilde{X}}_{I}^{(4)}, \tilde{\widetilde{Y}}_{V c}+\tilde{\widetilde{Y}}_{I}^{(4)}\right) e^{i k \alpha}\right] \\
& +\operatorname{Re}\left[\left(\widetilde{\widetilde{X}}_{I}^{(4)}, \widetilde{\widetilde{Y}}_{I}^{(4)}\right) e^{2 i k \alpha}\right],
\end{aligned}
$$

where $\tilde{X}_{V c}, \tilde{Y}_{V c}, \tilde{\tilde{X}}_{V c}$, and $\tilde{\tilde{Y}}_{V c}$ are obtainable from Eqs. (55)-(58), on substituting $\widetilde{x}_{I}, \widetilde{y}_{I}$, and $\widetilde{x}_{I}$ for $\tilde{x}, \tilde{y}$, and $\widetilde{x}$ in these expressions. The subscript $c$ is used to distinguish a variable of the core region.

With some algebra, one can show that

$$
\tilde{X}_{I}^{(4)}=\widetilde{\widetilde{X}}_{V c}=\widetilde{\widetilde{Y}}_{V c}=\widetilde{\widetilde{Y}}_{I}^{(4)}=0, \quad \text { and } \quad \widetilde{\widetilde{X}}_{I}^{(4)}=\text { constant } .
$$

Hence, the forcing comprises only second-order viscous interaction terms for the one-dimensional steady streaming and only fourth-order inviscid interaction terms for the cellular steady streaming. The $\exp (2 i k \alpha)$ forcing will only give rise to a steady pressure gradient, but not steady streaming of particles. We may accordingly decompose the time-mean variables into

$$
\begin{aligned}
\left(u, v, \bar{p}^{(4)}\right)= & \left(\widetilde{U}_{c}, 0, \widetilde{P}_{c}\right)+\operatorname{Re}\left[\left(\widetilde{\widetilde{U}}_{c}, \widetilde{\widetilde{V}}_{c}, \widetilde{\widetilde{P}}_{c}\right) e^{i k \alpha}\right] \\
& +\operatorname{Re}\left[\left(0,0, \widetilde{\widetilde{P}}_{c}\right) e^{2 i k \alpha}\right] .
\end{aligned}
$$

The one-dimensional Lagrangian drift in the core is governed by the same equation as Eq. (60):

$$
\nu \widetilde{U}_{c}^{\prime \prime}=-\tilde{X}_{V c}+\rho^{-1}\left(\widetilde{P}_{c}\right)_{\alpha},
$$

where $\left(\widetilde{P}_{c}\right)_{\alpha}$ is a constant. This equation can be solved in a similar manner as before:

$$
\begin{aligned}
\tilde{U}_{c}(\beta)= & E_{c}(\beta)-E_{c 0}+\tilde{U}_{b b l}^{\infty}+3\left[\tilde{U}_{b b l}^{\infty}+\tilde{U}_{t b l}^{\infty}-E_{c 0}-E_{c h}\right. \\
& \left.+2\left\langle E_{c}\right\rangle\right](\beta / h)^{2}-2\left[2 \tilde{U}_{b b l}^{\infty}+\tilde{U}_{t b l}^{\infty}-2 E_{c 0}-E_{c h}+3\left\langle E_{c}\right\rangle\right] \\
& \times(\beta / h), \\
& \rho^{-1}\left(\widetilde{P}_{c}\right)_{\alpha}=6 \nu h^{-2}\left[\tilde{U}_{b b l}^{\infty}+\tilde{U}_{t b l}^{\infty}-E_{c 0}-E_{c h}+2\left\langle E_{c}\right\rangle\right],
\end{aligned}
$$

where

$$
E_{c}(\beta)=\sigma k \operatorname{Re}\left\{\frac{1}{2}\left(|A|^{2}+|B|^{2}\right) \cosh 2 k \beta+A^{*} B \sinh 2 k \beta\right\},
$$

$$
\begin{aligned}
\left\langle E_{c}\right\rangle= & \sigma h^{-1} \operatorname{Re}\left\{\frac{1}{4}\left(|A|^{2}+|B|^{2}\right) \sinh 2 k h\right. \\
& \left.+\frac{1}{2} A^{*} B(\cosh 2 k h-1)\right\} .
\end{aligned}
$$

$E_{c 0}=E_{c}(0), E_{c h}=E_{c}(h), \widetilde{U}_{b b l}^{\infty}$, and $\widetilde{U}_{t b l}^{\infty}$ are given in Eqs. (91) and (92). by

The cellular Lagrangian drift in the core is now governed

$$
\begin{gathered}
\tilde{\widetilde{U}}_{c}=i k^{-1} \tilde{\widetilde{V}}_{c}^{\prime}, \\
\left(\frac{d^{2}}{d \beta^{2}}-k^{2}\right)^{2} \widetilde{\widetilde{V}}_{c}=\nu^{-1} k^{2}\left(\tilde{\widetilde{Y}}_{I}^{(4)}+i k^{-1} \widetilde{\widetilde{X}}_{I}^{(4) \prime}\right) .
\end{gathered}
$$

After some algebra, involving the use of Eqs. (111), (113), (117), (126), and (127), the right-hand side of the equation above can be worked out to be

$$
\begin{aligned}
\operatorname{RHS}(139)= & -\frac{1}{2} i \nu^{-1} \sigma^{2} k^{3} \widetilde{\bar{x}}_{I}^{*}\left(\widetilde{y}_{I}^{*} \tilde{y}_{I}^{\prime 2}+\widetilde{y}_{I} \widetilde{y}_{I}^{\prime} \widetilde{y}_{I}^{* \prime}\right)=M \sinh k \beta \\
& +N \cosh k \beta+Q \sinh 3 k \beta+R \cosh 3 k \beta,
\end{aligned}
$$

where

$$
\begin{aligned}
(M, N, Q, R)= & -\frac{1}{4} i \nu^{-1} \sigma^{2} k^{5} \widetilde{\widetilde{x}}_{I}^{*}\left[\left(A^{2} A^{*}-A^{*} B^{2}\right),\right. \\
& \left(-B^{2} B^{*}+A^{2} B^{*}\right),\left(A^{2} A^{*}+2 A B B^{*}+A^{*} B^{2}\right), \\
& \left.\left(B^{2} B^{*}+2 A A^{*} B+A^{2} B^{*}\right)\right] .
\end{aligned}
$$

The general solution to Eqs. (138) and (139) is 


$$
\begin{aligned}
\tilde{\widetilde{U}}_{c}(\beta)= & i F_{c} \cosh k \beta+i G_{c} k^{-1} \sinh k \beta+i G_{c} \beta \cosh k \beta \\
& +i H_{c} \sinh k \beta+i I_{c} k^{-1} \cosh k \beta+i I_{c} \beta \sinh k \beta \\
& +i k^{-1} \tilde{\widetilde{V}}_{c p}^{\prime}(\beta), \\
\tilde{\widetilde{V}}_{c}(\beta)= & F_{c} \sinh k \beta+G_{c} \beta \sinh k \beta+H_{c} \cosh k \beta \\
& +I_{c} \beta \cosh k \beta+\widetilde{\widetilde{V}}_{c p}(\beta),
\end{aligned}
$$

where the particular solution is

$$
\begin{aligned}
\tilde{\widetilde{V}}_{c p}(\beta)= & \left(8 k^{2}\right)^{-1}\left[M \beta^{2} \sinh k \beta+N \beta^{2} \cosh k \beta\right] \\
& +\left(64 k^{4}\right)^{-1}[Q \sinh 3 k \beta+R \cosh 3 k \beta] .
\end{aligned}
$$

On matching with the velocity components at the outer edge of the boundary layers, the constants $F_{c}-I_{c}$ can be determined as follows:

$$
\begin{gathered}
F_{c}=-i \tilde{\widetilde{U}}_{b b l}^{\infty}-k^{-1}\left[I_{c}+\tilde{\widetilde{V}}_{c p}^{\prime}(0)\right], \\
H_{c}=\tilde{\widetilde{V}}_{b b l}^{\infty}-\tilde{\widetilde{V}}_{c p}(0), \\
G_{c}=\left(g_{7} r_{3}-g_{8} r_{4}\right) /\left[\sinh ^{2} k h-(k h)^{2}\right], \\
I_{c}=\left(g_{7} r_{4}-g_{9} r_{3}\right) /\left[\sinh ^{2} k h-(k h)^{2}\right],
\end{gathered}
$$

where $g_{7}-g_{9}$ are given in Eq. (76), and

$$
\begin{aligned}
r_{3}= & k\left[\tilde{\widetilde{V}}_{t b l}^{\infty}+i \tilde{\widetilde{U}}_{b b l}^{\infty} \sinh k h-\tilde{\tilde{V}}_{b b l}^{\infty} \cosh k h+\tilde{\widetilde{V}}_{c p}(0) \cosh k h\right. \\
& \left.-\tilde{\widetilde{V}}_{c p}(h)\right]+\tilde{\widetilde{V}}_{c p}^{\prime}(0) \sinh k h, \\
r_{4}= & k\left[-i \tilde{\widetilde{U}}_{t b l}^{\infty}+i \tilde{\widetilde{U}}_{b b l}^{\infty} \cosh k h-\tilde{\widetilde{V}}_{b b l}^{\infty} \sinh k h+\tilde{\widetilde{V}}_{c p}(0) \sinh k h\right] \\
& +\tilde{\widetilde{V}}_{c p}^{\prime}(0) \cosh k h-\tilde{\widetilde{V}}_{c p}^{\prime}(h),
\end{aligned}
$$

in which $\tilde{\tilde{U}}_{b b l}^{\infty}, \quad \tilde{\widetilde{V}}_{b b l}^{\infty}, \quad \tilde{\widetilde{U}}_{t b l}^{\infty}$, and $\tilde{\widetilde{V}}_{t b l}^{\infty}$ are given in Eqs. (97)-(100).

Finally, the Lagrangian transport velocities which are uniformly valid across the whole fluid column are given by

$$
\tilde{U}(\beta)=\tilde{U}_{c}(\beta)+\tilde{U}_{b b l}(\beta)+\tilde{U}_{t b l}(\beta)-\tilde{U}_{b b l}^{\infty}-\tilde{U}_{t b l}^{\infty}
$$

for the one-dimensional steady streaming and

$$
\begin{aligned}
& \tilde{\widetilde{U}}(\beta)=\tilde{\widetilde{U}}_{c}(\beta)+\widetilde{\widetilde{U}}_{b b l}(\beta)+\widetilde{\widetilde{U}}_{t b l}(\beta)-\tilde{\widetilde{U}}_{b b l}^{\infty}-\widetilde{\widetilde{U}}_{t b l}^{\infty}, \\
& \tilde{\widetilde{V}}(\beta)=\tilde{\widetilde{V}}_{c}(\beta)+\tilde{\widetilde{V}}_{b b l}(\beta)+\tilde{\widetilde{V}}_{t b l}(\beta)+i k{\widetilde{\tilde{U}_{b b l}}}_{b-} \beta-\widetilde{\widetilde{V}}_{b b l}^{\infty}+i k \widetilde{\tilde{U}}_{t b l}^{\infty}(\beta \\
& -h)-\tilde{\widetilde{V}}_{t b l}^{\infty}
\end{aligned}
$$

for the cellular steady streaming.

\section{DISCUSSIONS}

The problems and expressions deduced above can be nondimensionalized using the following normalization scheme, where the normalized quantities are distinguished by an overhead caret:

$$
\begin{aligned}
& \left(\hat{b}, \hat{A}, \hat{B}, \hat{C}, \hat{D}, \hat{x}^{(1)}, \hat{y}^{(1)}, \hat{\tilde{x}}, \hat{\tilde{y}}, \hat{\tilde{\tilde{x}}}\right)=\left(b, A, B, C, D, x^{(1)}, y^{(1)}, \widetilde{x}, \tilde{y}, \widetilde{\tilde{x}}\right) / a, \\
& (\hat{\alpha}, \hat{\beta}, \hat{\delta})=(\alpha, \beta, \delta) / h, \quad(\hat{k}, \hat{\lambda}, \hat{s})=(k, \lambda, s) h, \\
& \left(\hat{p}^{(1)}, \hat{\tilde{p}}, \hat{\tilde{\tilde{p}}}\right)=\left(p^{(1)}, \tilde{p}, \tilde{\tilde{p}}\right) /\left(\rho \sigma^{2} a h\right), \\
& \left(\hat{u}, \hat{v}, \hat{\tilde{U}}, \tilde{\widetilde{U}}, \hat{\widetilde{\tilde{V}}}, \hat{\widetilde{V}}_{p}, \hat{E}, \hat{F}, \hat{H}\right)=\left(u, v, \widetilde{U}, \widetilde{\widetilde{U}}, \widetilde{\widetilde{V}}, \widetilde{\widetilde{V}}_{p}, E, F, H\right) /\left(\sigma a^{2} / h\right), \\
& (\hat{G}, \hat{I})=(G, I) /\left(\sigma a^{2} / h^{2}\right), \quad\left(\hat{\bar{p}}^{(2)}, \hat{\widetilde{P}}, \hat{\tilde{P}}\right)=\left(\bar{p}^{(2)}, \widetilde{P}, \widetilde{\widetilde{P}}\right) /\left(\rho \sigma^{2} a^{2}\right), \\
& (\hat{M}, \hat{N}, \hat{Q}, \hat{R})=(M, N, Q, R) /\left(\sigma a^{2} / h^{5}\right) .
\end{aligned}
$$

The primary variables of interest are the second-order timemean variables $\hat{u}$ and $\hat{v}$, which are functions of $(\hat{\alpha}, \hat{\beta})$. The input parameters are the wavelength $\hat{k}$, the upper wave amplitude/phase $\hat{b}$, the oscillation frequency $\hat{\delta}$, and the bottom wave amplitude $\epsilon=a / h$.

Our frequency parameter $\hat{\delta}$ is the ratio of the Stokes layer thickness to the channel depth; the higher the frequency, the smaller the parameter. This parameter can be written as $\hat{\delta}$ $=\sqrt{2} \Lambda^{-1}$, where $\Lambda=h(\sigma / \nu)^{1 / 2}$ is the well-known Womersley's number often used in oscillatory physiological flows. The steady-streaming Reynolds number is here defined to be $\operatorname{Re}_{s} \equiv(a / \delta)^{2}=(\epsilon / \hat{\delta})^{2}$. We reiterate that the results presented in Sec. IV are good for small or large $\hat{\delta}$ as long as $\operatorname{Re}_{s} \ll 1$ or $a \ll \delta$, while those presented in Sec. V are valid for $\hat{\delta} \ll 1$ and $\mathrm{Re}_{s}=O(1)$ or $a \sim \delta$. We also remark that the normalization scale for the second-order velocities is $\sigma a^{2} / h=2 \operatorname{Re}_{s}(\nu / h)$, and hence the basic dependence of these velocities on $\mathrm{Re}_{s}$ is 


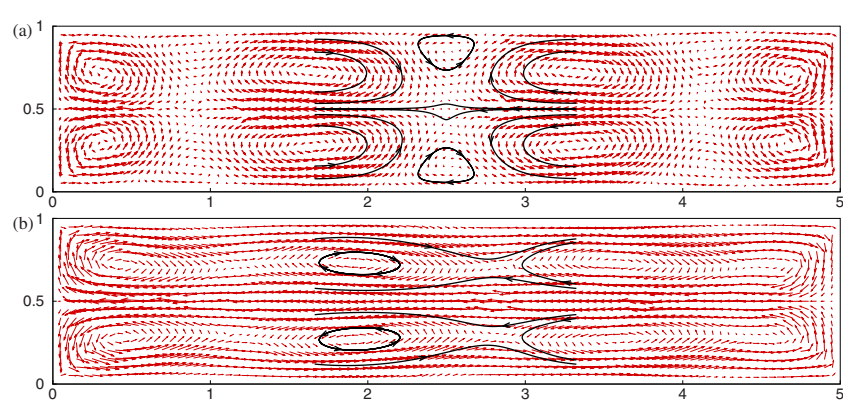

FIG. 2. (Color online) Steady Lagrangian flow fields as generated by the present analytical model (streamlines) and the present numerical simulation by FLUENT (vectors), which compare favorably with (a) Fig. 7 and (b) Fig. 8 of Yi et al. [6]. In these cases, $L=5 h=3 \ell, \hat{k}=3.77, \epsilon=0.01$, and (a) $\hat{\delta}=1.41$, (b) $\hat{\delta}=0.18$.

already taken into account by the normalization. In the normalized problems, the only place where $\operatorname{Re}_{s}$ shows up further is a linear factor on the left-hand side of Eq. (139) and hence a linear factor of the particular solution Eq. (144). Therefore, when normalized, only the cellular steady-streaming component under fast oscillation is affected by the steady-streaming Reynolds number. The normalized one-dimensional steadystreaming component is independent of $\operatorname{Re}_{s}$ under all conditions.

To check the accuracy of our theories presented above, we have generated results to compare with previously published cases including the analytical results presented in Fig. 10 of Selverov and Stone [5] and the numerical results presented in Figs. 7-9 of Yi et al. [6]. Our results are found to be in very good agreement with these published results. For illustration, we show in Fig. 2 the steady Lagrangian flow fields that we have generated in order to compare with the cases presented in Figs. 7 and 8 of Yi et al. [6]. In these plots, in addition to the analytical results (streamlines), we show also numerical results of our own (vectors). These numerical results, which provide further independent checking of our analytical model, were produced using the computational fluid dynamics package FLUENT. To handle the moving boundaries, the spring-based smoothing method of dynamic meshing in FLUENT was employed. On performing the numerical simulations, we carried out the computation for ten wave periods to allow the transients to die out before hundreds of discrete particles were evenly injected into the flow field to get their net displacements over a short period of time. To get the particle trajectories, the discrete phase model in FLUENT was applied. Very fine particles were used in order to avoid the inertia effect. A time step equal to one-hundredth of a wave period was used. The vectors in Fig. 2 are the net displacements of the particles over one wave period of time, which also represent the steady Lagrangian transport field. One can readily check that both our analytical and simulation results are in agreement with the numerical results presented by $\mathrm{Yi}$ et al. [6].

One of the primary concerns of the present problem is the mixing induced by the Lagrangian transport. The transport is largely advection in nature and, hence, is closely dependent on the magnitude of the velocity. It is desirable if some kind of a parameter can be used to characterize the overall mixing

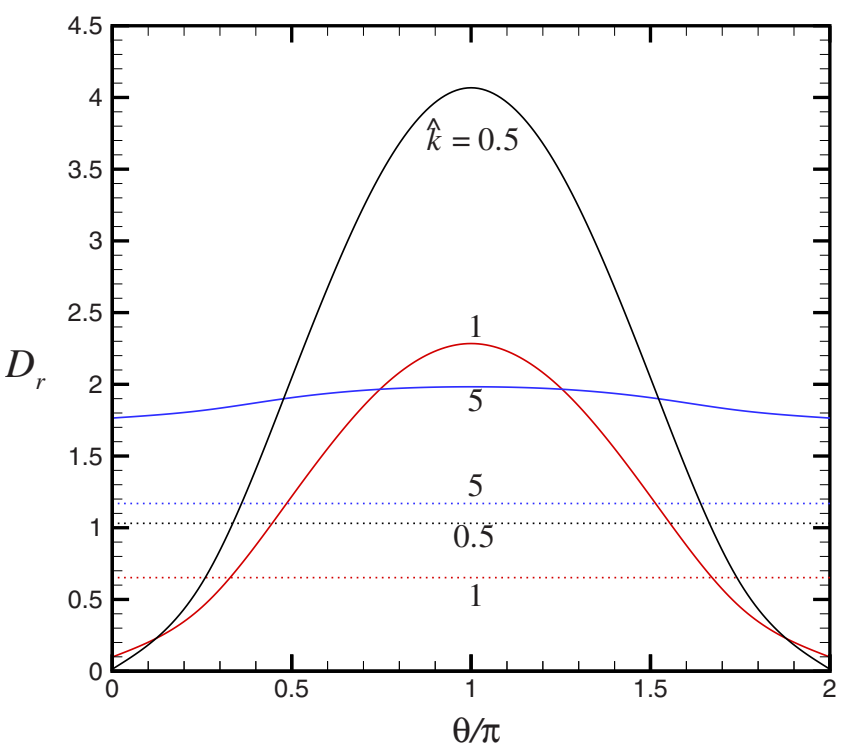

FIG. 3. (Color online) The mean drift parameter $D_{r}$ as a function of the phase shift between the upper and lower waves $\theta$ and wave number $\hat{k}$, where the solid and dotted lines are for $|\hat{b}|=1$ and $\hat{b}=0$, respectively, and $\hat{\delta}=\epsilon=0.01$.

performance of a flow field. Here, we have chosen to use the spatial-averaged magnitude of the mass transport velocity as a parameter to represent the gross advective strength of the flow field. For simplicity, let us call this parameter, defined below, the mean drift parameter:

$$
D_{r}=\frac{\hat{k}}{2 \pi} \int_{0}^{1} \int_{0}^{2 \pi / \hat{k}}\left(\hat{u}^{2}+\hat{v}^{2}\right)^{1 / 2} d \hat{\alpha} d \hat{\beta} .
$$

Let us now look into various effects on this mean drift parameter. Except stated otherwise, the values $\hat{\delta}=0.01$ and $\epsilon$ $=0.01$ are considered in the calculations discussed below.

The amplitude of the wave traveling on the upper wall can be written as

$$
\hat{b}=|\hat{b}| e^{i \theta},
$$

where $\theta$ is the phase difference between the waves on the two walls. In Fig. 3, we show the mean drift parameter $D_{r}$ as a function of $\theta$ and $\hat{k}$ for $|\hat{b}|=1$. The corresponding values for $|\hat{b}|=0$ are also plotted for comparison. Obviously, for any wavelength, the drift parameter is the minimum when $\theta=0$ (i.e., the waves are in phase) and is maximum when $\theta=\pi$ (i.e., the waves are $180^{\circ}$ out of phase). As pointed out already, when the two waves are in phase such that $\hat{b}=1$, the steady Lagrangian flow field is purely one dimensional. In the absence of cellular flow, the overall transport strength is the weakest. On the contrary, when $\hat{b}=-1$ or the waves on the two walls are symmetric about the channel axis, the overall flow field is the strongest. When $\hat{k} \leq 1$ (i.e., long waves), the drift parameter varies significantly with the phase; the maximum at $\theta=\pi$ is much larger than the minimum at $\theta$ $=0$. Also, the maximum value is approximately four times the value for $|\hat{b}|=0$ implying that the drift (which is a 


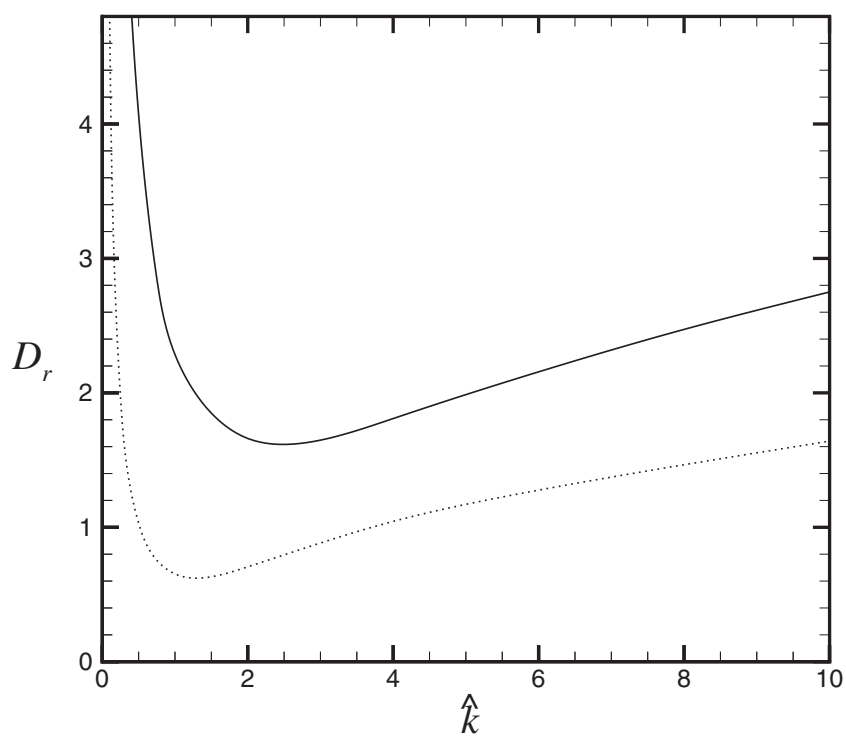

FIG. 4. The mean drift parameter $D_{r}$ as a function of the wave number $\hat{k}$, where the solid and dotted lines are for $\hat{b}=-1$ and $\hat{b}=0$, respectively, and $\hat{\delta}=\epsilon=0.01$.

second-order quantity proportional to $a^{2}$ ) due to two equal waves of $\pi$-phase shift on two walls is grossly the same as that due to a wave with twice the amplitude on one wall only. The dependence on the phase diminishes as $\hat{k}$ gets large. For $\hat{k} \gg 1$ (i.e., short waves), the drift parameter becomes constant irrespective of the phase shift. The value is, however, smaller than four times that for $|\hat{b}|=0$. Hence, for short waves, it appears to be less advantageous to impose two waves than to impose one wave with twice the amplitude.

The effect of the wave number $\hat{k}$ on the drift parameter $D_{r}$ is further shown in Fig. 4, for $\hat{b}=-1,0$. The relationship is nonmonotonous. For any set of parameters, the drift parameter reaches a minimum at a point where $\hat{k}=O(1)$. The parameter increases on approaching either the long or short wave limits. Figures 5-7 show the steady Lagrangian flow field as represented by streamlines for three values of the wave number $\hat{k}=0.5,1.0,5.0$, where $\hat{b}=0$ (i.e., only one wave applied on the channel bottom). Under long waves such that $\hat{k}<1$, two recirculating cells of comparable strength are formed within one wavelength. Hence, with long waves, mixing is largely accomplished through the recirculating motions of the two cells, which take place within one wavelength. There is a region in $0<\hat{\alpha}<2$ where the streaming velocity is relatively weak and is practically a stagnant zone. Hence, individual wavelengths are separated by stagnant zones, which tend to retard mixing across the entire channel. As $\hat{k}$ increases to become order unity, both cells diminish in their strength, particularly the upper one. As $\hat{k}$ further increases such that the wave becomes very short or $\hat{k} \gg 1$, the upper cell vanishes completely, while the lower one is much reduced in size. The entire streaming flow field is dominated by the one-dimensional component. The forward mass transport, which is with high velocity, is through a narrow passage underneath the recirculating cell near the

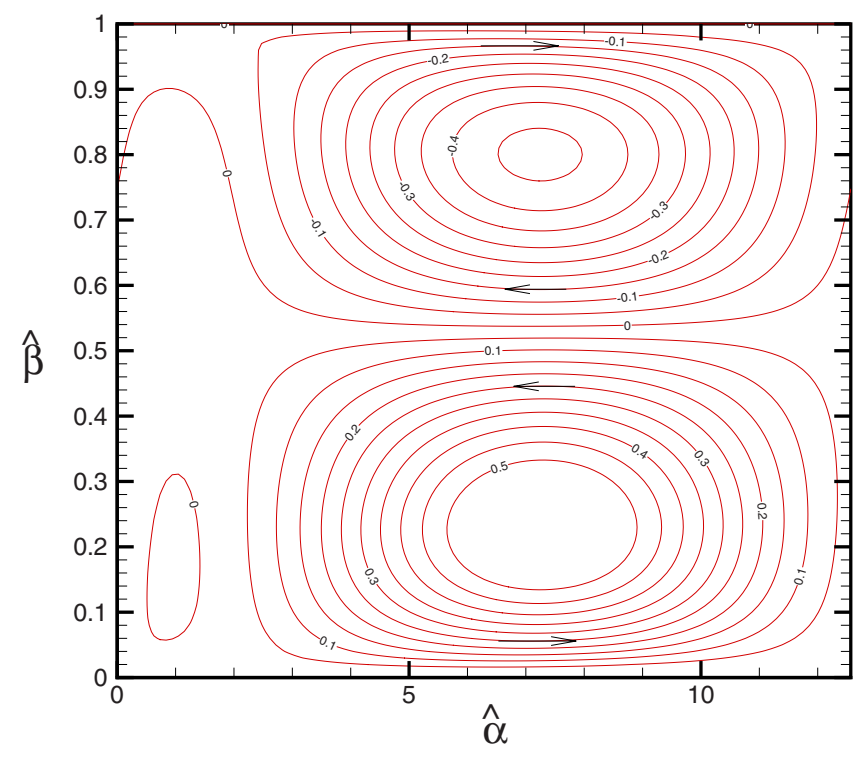

FIG. 5. (Color online) Streamlines for the steady Lagrangian flow field, where $\hat{k}=0.5, \hat{b}=0, \hat{\delta}=0.01$, and $\epsilon=0.01$.

bottom of the channel. The backward mass transport, which is with lower velocity, is through a wider passage above the cell. Hence, under short waves, the mixing is largely accomplished through the recirculation across the entire channel between the two sidewalls.

The results being not shown here, we have found that the drift parameter will in general decrease with increasing $\hat{\delta}$ (i.e., slower oscillation). Hence, to achieve high mixing performance, the peristaltic pumping must be operated at a sufficiently high frequency so that $\hat{\delta} \ll 1$. We have also found that the drift parameter will not be much affected by $\epsilon$ as long as the steady-streaming Reynolds number $\operatorname{Re}_{s} \leq O(1)$. The steady-streaming flow field will, however, be affected by this wave amplitude parameter. Figure 8 shows the flow field

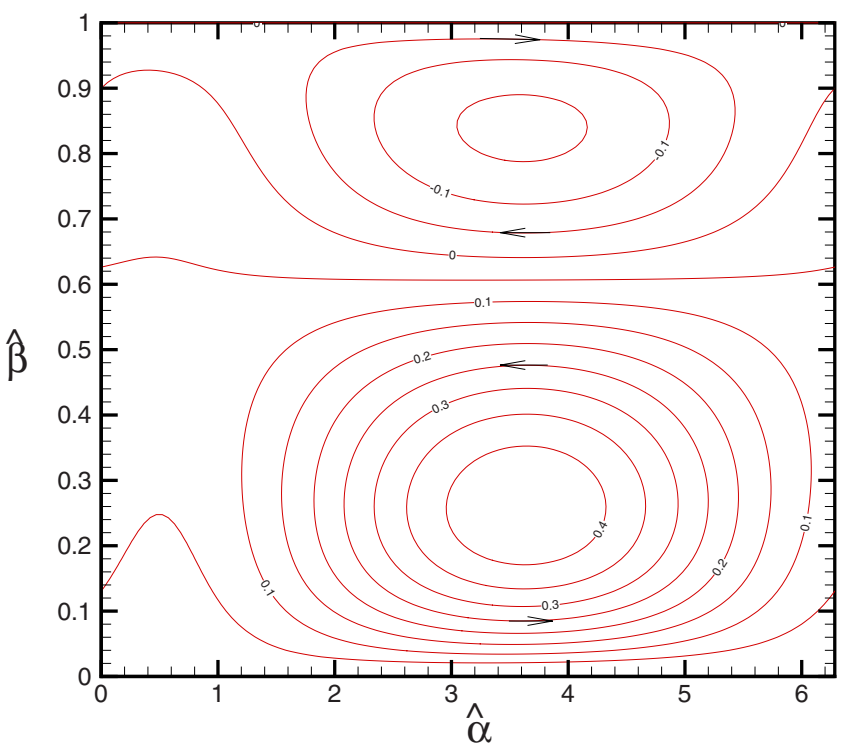

FIG. 6. (Color online) Streamlines for the steady Lagrangian flow field, where $\hat{k}=1.0, \hat{b}=0, \hat{\delta}=0.01$, and $\epsilon=0.01$. 


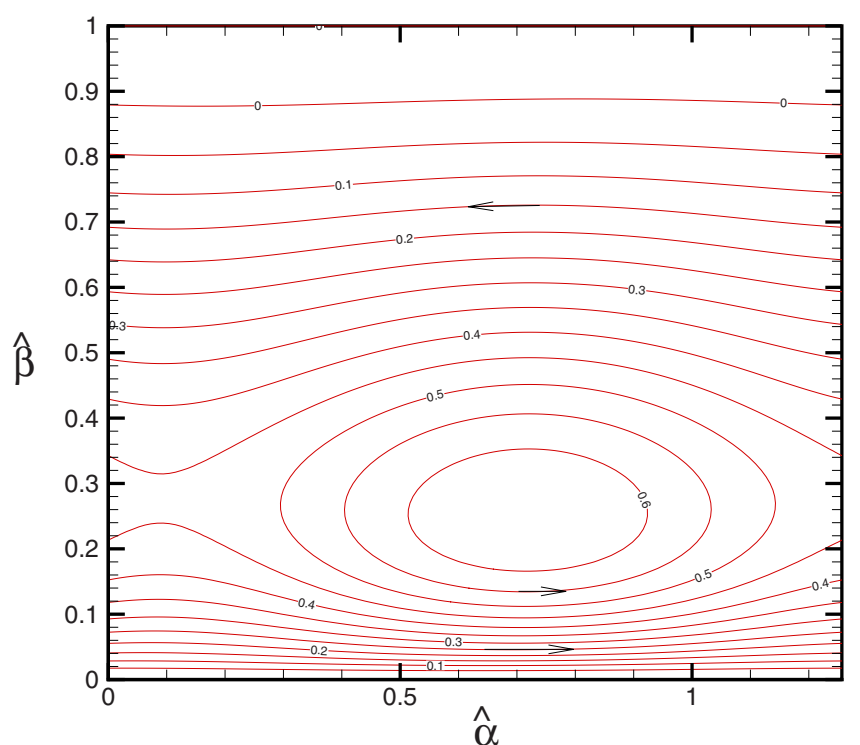

FIG. 7. (Color online) Streamlines for the steady Lagrangian flow field, where $\hat{k}=5.0, \hat{b}=0, \hat{\delta}=0.01$, and $\epsilon=0.01$.

for a higher value of wave amplitude $\epsilon=0.1$, for $\hat{k}=0.5$ and $\hat{b}=0$. When this figure is compared with Fig. 5, one can see an obvious change in pattern of the two recirculating cells upon increasing $\epsilon$ from 0.01 to 0.1 . With a larger-amplitude wave being applied on the bottom, the lower cell has become bigger in size, and as a result, the stagnant zone is much reduced in size. This will lead to the much desired mixing across the entire channel in addition to that within individual wavelengths.

\section{CONCLUDING REMARKS}

In this paper, we have examined the steady Lagrangian flow field induced by small-amplitude peristaltic waves applied to the boundaries of a two-dimensional closed cavity. Here, the problem is entirely Lagrangian in formulation and analysis. Under the general condition that the Stokes layer thickness is comparable with the channel depth, which is much greater than the wave amplitude, the steady-streaming velocities are given in Eq. (59), where the one-dimensional component is given in Eq. (61), and the cellular components are given in Eqs. (68) and (69). This is the case that has been considered previously by Selverov and Stone [5] and Yi et al. [6]. These authors, however, failed to fully present their so-

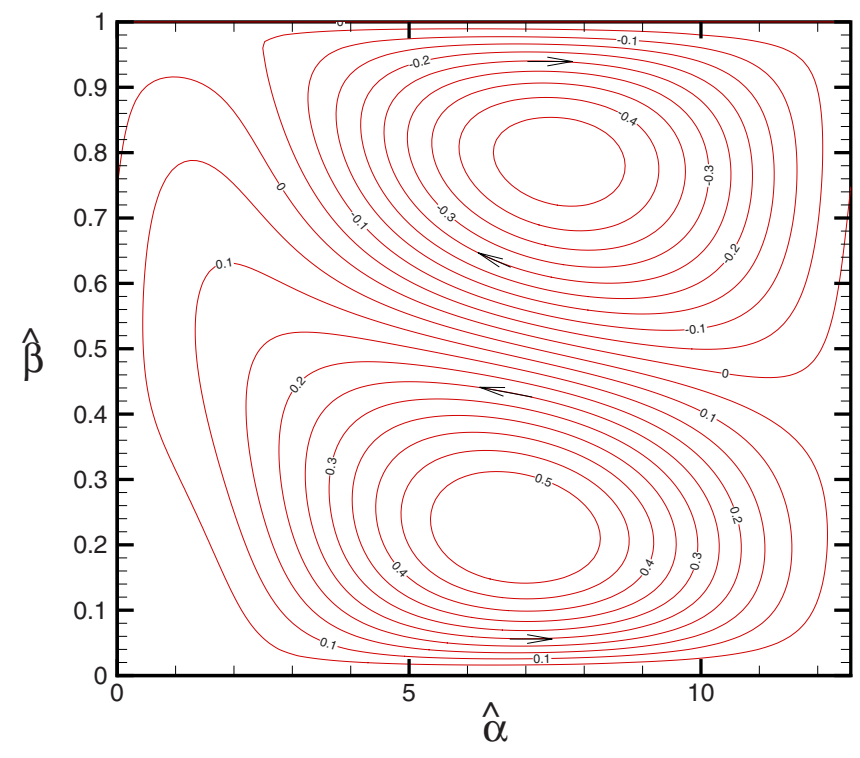

FIG. 8. (Color online) Streamlines for the steady Lagrangian flow field, where $\hat{k}=0.5, \hat{b}=0, \hat{\delta}=0.01$, and $\epsilon=0.1$.

lutions owing to the complexity in their expressions. We have also found analytically the velocities, as given in Eqs. (151)-(153), for the more important case when the frequency is so high that the Stokes layer thickness becomes comparable with the wave amplitude. In summary, our model is purely analytical and is valid to $\operatorname{Re}_{s}=O(1)$ yielding directly Lagrangian drift velocities with simple and straightforward calculations. This distinguishes our work from those in the literature.

We have also looked into the mixing effect of the Lagrangian transport under various configurations. If waves are applied to both boundaries, the two waves should be at a phase shift of $\pi$ to result in the maximum possible transport rate. Otherwise, it will be more desirable if one wave of twice the amplitude is applied to one of the boundaries. The other conditions conducive to mixing are long wavelength $\hat{k} \ll 1$, high frequency $\hat{\delta} \ll 1$, and relatively large amplitude $\epsilon / \hat{\delta}>1$.

\section{ACKNOWLEDGMENT}

The work was supported by the Research Grants Council of the Hong Kong Special Administrative Region, China through Project No. HKU 7156/09E.
[1] C. Barton and S. Raynor, Bull. Math. Biophys. 30, 663 (1968).

[2] Y. C. Fung and C. S. Yih, Trans. ASME, J. Appl. Mech. 35, 669 (1968).

[3] M. Y. Jaffrin and A. H. Shapiro, Annu. Rev. Fluid Mech. 3, 13 (1971).

[4] F. C. P. Yin and Y. C. Fung, J. Fluid Mech. 47, 93 (1971).
[5] K. P. Selverov and H. A. Stone, Phys. Fluids 13, 1837 (2001).

[6] M. Yi, H. J. Bau, and H. Hu, Phys. Fluids 14, 184 (2002).

[7] F. Carlsson, M. Sen, and L. Löfdahl, Phys. Fluids 16, 1822 (2004).

[8] F. Carlsson, M. Sen, and L. Löfdahl, Eur. J. Mech. B/Fluids 24, 366 (2005).

[9] K. J. Lee, I. Shugan, and K. H. Kim, J. Korean Phys. Soc. 45, 
399 (2004).

[10] S. Kumar, H. J. Kim, and A. Beskok, ASME J. Fluids Eng. 129, 1361 (2007).

[11] X. Mandviwalla and R. Archer, ASME J. Fluids Eng. 130, 124501 (2008).
[12] M. S. Longuet-Higgins, Philos. Trans. R. Soc. London, Ser. A 245, 535 (1953).

[13] W. J. Pierson, J. Geophys. Res. 67, 3151 (1962).

[14] Ü. Ünlüata and C. C. Mei, J. Geophys. Res. 75, 7611 (1970). 